TITLE:

\title{
Dynamics near QCD critical point by dynamic renormalization group
}

AUTHOR(S):

Minami, Yuki

CITATION:

Minami, Yuki. Dynamics near QCD critical point by dynamic

renormalization group. Physical Review D 2011, 83(9): 094019.

ISSUE DATE:

2011-05

URL:

http://hdl.handle.net/2433/145973

RIGHT:

(C) 2011 American Physical Society. 
PHYSICAL REVIEW D 83, 094019 (2011)

\title{
Dynamics near QCD critical point by dynamic renormalization group
}

\author{
Yuki Minami* \\ Department of Physics, Kyoto University, Kyoto 606-8502, Japan
}

(Received 14 March 2011; published 13 May 2011)

\begin{abstract}
We work out the basic analysis on dynamics near the QCD critical point $(C P)$ by the dynamic renormalization group ( $\mathrm{RG}$ ). In addition to the RG analysis by coarse-graining, we construct the nonlinear Langevin equation as a basic equation for the critical dynamics. Our construction is based on the generalized Langevin theory and the relativistic hydrodynamics. Applying the dynamic RG to the constructed equation, we derive the RG equation for the transport coefficients and analyze their critical behaviors. We find that the resulting RG equation turns out to be the same as that for the liquid-gas $C P$ except for an insignificant constant. Therefore, the bulk viscosity and the thermal conductivity strongly diverge at the QCD $C P$. We also show that the thermal and viscous diffusion modes exhibit critical slowing down with the dynamic critical exponents $z_{\text {thermal }} \sim 3$ and $z_{\text {viscous }} \sim 2$, respectively. In contrast, the sound propagating mode shows critical speeding up with the negative exponent $z_{\text {sound }} \sim-0.8$.
\end{abstract}

DOI: 10.1103/PhysRevD.83.094019

PACS numbers: $12.38 .-\mathrm{t}, 05.70 . J k, 25.75 . \mathrm{Nq}$

\section{INTRODUCTION}

An interesting feature of the phase diagram in quantum chromodynamics (QCD) is the possible existence of the critical point $(C P)$, which is predicted by various effective models of QCD and suggested by lattice QCD simulations. The critical point is the end point of the first order phase transition line existing in the low temperature $(T)$ region [1]. ${ }^{1}$ Then, the significance of this QCD $C P$ is that the phase transition at this point is of second order, and thereby we can expect critical phenomena due to large fluctuations of various quantities at this point [8].

Then, a fundamental problem arises: what is the soft mode of the QCD $C P$ ? A hint is that the baryon-number susceptibility [9] diverges at the $C P$ as first suggested in [9] and is subsequently demonstrated in [10]. It has been established that the fluctuating modes of conserved densities are the soft modes at the $C P[11,12]$. Although the $\sigma$ mode seems to be the soft mode, it couples with the density fluctuation at finite density [9] and remains massive $[11-13] .^{2}$

Furthermore, as a critical phenomenon, some authors suggested a divergent behavior of bulk viscosity at the QCD $C P$ [15], although the validity of their argument is very much controversial [16-18]; for instance, the ansatz for the spectral function adopted in [15] may not necessarily be true [16], and a microscopic calculation by the relativistic Boltzmann equation [18] shows that the

\footnotetext{
*Electronic address: y-minami@ruby.scphys.kyoto-u.ac.jp

${ }^{1}$ In fact, the QCD matter seems to have an extremely rich structure in the phase diagram with one or multiple critical points [1-3], and even accompanied with inhomogeneous phases at low $T[4,5]$, although the very existence of a $C P$ may be questioned according to [6,7].

${ }^{2}$ Such a fast mode as the $\sigma$ near the QCD $C P$ is called a slaved mode [14], because its slow dynamics is controlled by the density fluctuation for the QCD $C P$.
}

bulk viscosity is finite at the $C P$. Thus, it is still not obvious whether the transport coefficients will diverge at the QCD $C P$.

In fact, as is known in condensed matter physics, the critical divergence of the transport coefficients is a common phenomenon at a $C P$, such as at the liquid-gas $C P$, and originate from a universal mechanism; nonlinear fluctuations of macroscopic variables cause the divergence $[19,20]$. This implies that microscopic processes, as may be described by such as the Boltzmann equation, would give only a minor contribution to the critical divergence of these quantities, if any. The dynamic renormalization group (RG) theory [21,22] is a standard technique for critical dynamics, which systematically incorporate the macroscopic fluctuations causing the divergent behavior of transport coefficients. In this theory, we must construct a nonlinear Langevin equation as a basic equation for the critical dynamics. The construction goes as follows. First, the slow variables are identified for describing the critical dynamics. Next, the thermodynamic potential for the slow variables is constructed to determine the static property of the system. Finally, the streaming terms, causing the dynamic-nonlinear coupling, and the kinetic coefficients are determined for, respectively, describing time-reversible and irreversible changes of the slow variables. We note that the streaming term is absent in the simple Brownian motion.

The general theory of the critical dynamics as described above tells us that an essential ingredient is to properly construct the nonlinear Langevin equation for the critical dynamics. As far as we know, this is the first attempt for the QCD CP. Our construction of the Langevin equation is based on the generalized Langevin theory, by Mori [19,23], and the relativistic hydrodynamics, because the slow variables are identified as long-wavelength fluctuations of the conserved densities [11,12,24]; we construct the streaming terms from continuity equations and the 
potential condition, which is a general condition for streaming terms [21,22]. Also, we use the thermodynamic potential for the $3 \mathrm{D}$ Ising system as that for the QCD $C P$ because the static universality class is the same as the 3D Ising class [11,12,25]. Finally, we determine the kinetic coefficients from a relativistic hydrodynamic equation; here the so-called Landau equation [26] is taken. In consequence, we shall show that the Langevin equation differs from it for the liquid-gas $C P$ due to relativistic effects, although the dynamic universality class of the QCD $C P$ is conjectured as of the liquid-gas $C P[12,17]$.

After such construction, we apply the dynamic RG to the Langevin equation and derive the $R G$ equations for the transport coefficients. Consequently, to our surprise, these $\mathrm{RG}$ equations turn out to be the same as for the liquid-gas $C P$ except for a irrelevant constant, although the Langevin equations are different. Therefore, the bulk viscosity and the thermal conductivity strongly diverge and can be more important than the shear viscosity near the QCD $C P$. We shall also show that the thermal and viscous diffusion modes exhibits critical slowing down, whereas the sound mode critical speeding up.

This paper is organized as follows. In Sec. II, we shortly review the theory of critical dynamics. In Sec. III, we construct the nonlinear Langevin equation for the QCD $C P$ by the generalized Langevin theory and the relativistic hydrodynamics. In Sec. IV, we analyze the critical exponents of the transport coefficients and dynamic critical exponents by the dynamic RG. The final section is devoted to a summary and concluding remarks. In Appendixes A, $B$, and $C$, we give the detailed derivation of the $R G$ equations for the transport coefficients.

\section{REVIEW OF CRITICAL DYNAMICS}

Since the present work is based on the theory of critical dynamics in condensed matter physics [21,22], we now shortly review it for self-containedness.

\section{A. Critical divergences of transport coefficients}

The critical divergence of transport coefficients (or diffusion constants) is a common phenomenon, for instance, at the critical point of the liquid-gas, ferromagnetic transitions and so on $[21,22]$. The important point is that the critical divergence originates from a universal mechanism; nonlinear fluctuations of macroscopic variables causes the divergence [19,20].

Here, we briefly illustrate how macroscopic nonlinear fluctuations cause the critical divergence, taking the thermal conductivity near the liquid-gas $C P$ as an example [27]. The thermal conductivity is given by the Kubo formula as follows,

$$
\lambda=T^{-2} \int d \mathbf{r} \int_{0}^{\infty} d t\langle q(\mathbf{r}, t) q(0,0)\rangle,
$$

where $q(\mathbf{r}, t)$ and $T$ are the heat current and temperature, respectively, and $\langle\cdots\rangle$ denotes the statistical average in the thermal equilibrium state. The heat current $q(\mathbf{r}, t)$ is supplied from two sources: one is due to a microscopic process as calculated by a microscopic theory, such as the Boltzmann equation, and the other is due to nonlinear fluctuations of macroscopic variables [23]:

$$
q=q_{\text {micro }}+q_{\text {macro }}
$$

where $q_{\text {micro }}$ and $q_{\text {macro }}$ respectively denote the microscopic and macroscopic currents. The macroscopic process causing the heat current is identified as the entropy density convected by fluid velocity fluctuation. Thus, we have

$$
q_{\text {macro }} \sim \delta s \delta v,
$$

where $\delta s$ and $\delta v$ respectively denote the fluctuations of the entropy density and the fluid velocity. The macroscopic current Eq. (3) is of the second order in fluctuations and hence negligible far from the $C P$. However, it becomes the dominant part near the $C P$, since the fluctuations are enhanced there. We see that Eq. (1) now has the following form:

$$
\lambda=\lambda_{\text {micro }}+\int d \mathbf{r} \int_{0}^{\infty} d t\langle\delta s(\mathbf{r}, t) \delta v(\mathbf{r}, t) \delta s(0,0) \delta v(0,0)\rangle
$$

where $\lambda_{\text {micro }}$ is the thermal conductivity coming from the microscopic current. Recalling that the entropy density fluctuation is a soft mode near the liquid-gas $C P$, we see that the second term of Eq. (4) diverges at the $C P$. This is the mechanism causing the critical divergence of transport coefficients.

Let us call the transport coefficients, such as $\lambda_{\text {micro }}$, coming from microscopic processes the bare transport coefficients, and those including the contributions from the nonlinear macroscopic fluctuations the renormalized ones. Then, we need not to study the critical divergence of transport coefficients by a microscopic theory since the divergence originates from only the macroscopic processes. The dynamic RG [21,22,25,28,29] is the standard theory incorporating such nonlinear macroscopic fluctuations. In this theory, we must construct a nonlinear Langevin equation as a basic equation for the critical dynamics.

\section{B. Generalized Langevin equation}

We first note that if the dynamic variables are divided into slow and fast ones, the slow dynamics can be well described by a Langevin equation. We stress that such the Langevin equation can be derived in a generic way, so called the Mori theory [19,23], from the microscopic equation of motion. The starting microscopic equations of motion are Liouville or Hisenberg equation for a classical or a quantum system, respectively. They read 


$$
\frac{\partial}{\partial t} A_{j}(t)=\left\{A_{j}(t), H\right\}_{\mathrm{PB}},
$$

and

$$
\frac{\partial}{\partial t} A_{j}(t)=(1 / i \hbar)\left[A_{j}(t), H\right],
$$

respectively. Here, $A_{j}(t)$ are arbitrary slowly varying variables (slow variables), $H$ a microscopic Hamiltonian, and, $\{\cdot, \cdot\}_{\mathrm{PB}}$ and $[\cdot, \cdot]$ represent Poisson brackets and commutation relations, respectively. Equation (5) (or Eq. (6)) can be divided into two parts: one is composed of only the slow variables and describes their slow motion, while the other involves fast motions due to the microscopic processes. Thus, we have [19,21]

$$
\frac{\partial}{\partial t} A_{j}(t)=v_{j}(A)-\sum_{k} L_{j k}(A) \frac{\delta(\beta H(A))}{\delta A_{k}}+\theta_{j}(t),
$$

with $\beta$ being the inverse temperature. Here, $v_{j}(A(t))$, which is called a streaming term, gives the time-reversible process, while $L_{j k}$ and $H(A(t))$ are bare kinetic coefficients and a thermodynamic potential for $A_{j}$, respectively. The first and second terms in Eq. (7) are the slow motions and nonlinear in $A_{j}$, whereas the last term is the fast motions and treated as a stochastic variable obeying the fluctuationdissipation relation

$$
\left\langle\theta_{j}(t) \theta_{k}\left(t^{\prime}\right) ; a\right\rangle=2 L_{j k}(a) \delta\left(t-t^{\prime}\right) .
$$

Here, $\langle\cdots ; a\rangle$ represents the conditional average in which $A_{j}$ is fixed at $a_{j}$. We stress that this relation is not given by hand, but naturally obtained from the decomposition process [19].

Equation (7) is called the generalized Langevin equation, which has been widely used in the phase transition dynamics [21,22]. Even for the QCD $C P$, we may use the generalized Langevin equation, because only the time scale separation is assumed in the Mori theory. Furthermore, we note that, by the time scale separation, transport coefficients arises.

\section{Dynamic RG}

The general dynamic RG transformation usually consists of two procedures, i.e., coarse-graining and rescaling as in the static RG transformation [22,30]. However, as is shown in $[21,25,29]$, we can omit the rescaling, if we are interested in only the critical exponents of transport coefficients, although the relevant-fixed point seems to be absent in such a simplified RG transformation [29].

The Langevin equation is an infrared effective theory and inherently has an ultraviolet cutoff $\Lambda_{0}$, which should satisfy the following inequality

$$
\xi^{-1} \ll \Lambda_{0} \ll a^{-1} .
$$

Here, $\xi$ and $a$ are the correlation length and the characteristic microscopic length, respectively. Then, the Langevin equation is coarse-grained by averaging over the large wave number components of the slow variables $A_{j}(t)$ in the infinitesimal wave number shell,

$$
\Lambda-\delta \Lambda<k<\Lambda \text {, }
$$

for Eq. (7). Here, $\Lambda$ starts from the initial value $\Lambda_{0}$ and is lowered up to $\Lambda \ll \xi^{-1}$. In this way, we infinitesimally make coarse-graining of the Langevin equation. Because the coarse-graining procedure is infinitesimal, we do not need the rescaling. Inspecting the form of the coarsegrained Langevin equation, we can obtain the RG equation for the transport coefficients.

\section{Contrast with the static RG}

Here, we first stress that the concept of the dynamic universality class is not so universal contrary to its name. Then, the class of the QCD CP may not be the same as of the liquid-gas $C P$ or the model $H,{ }^{3}$ although it is conjectured by $[12,17]$. To see this, let us contrast the difference between the static RG with the dynamic one.

An important point is that the respective infrared effective theories are different; in the static case, the infrared effective theory is the thermodynamic potential (or socalled Landau free energy), the nature of which turns out to be governed by only the space dimension and the symmetry among the order parameters but not by the details of the dynamics, and hence the concept of the universality class makes sense for the static case. In contrast, for the dynamic RG, the infrared effective theory is the nonlinear Langevin equation.

Here, the important difference arises. The relevant variables for the Langevin equationare not only the order parameters but also conserved densities, and its nonlinear couplings cannot be determined by only the symmetry in general. Consequently, the dynamic universality class is not so universal compared to the static one. Specifically, the nonlinear couplings, namely the streaming terms $v_{j}(A)$, are generally given by the Poisson brackets (commutation relations) among the slow variables in the classical (quantum) system [22]:

$$
v_{j}(A)=\sum_{k}\left[Q_{j k}(A) \frac{\delta H}{\delta A_{k}}-\beta^{-1} \frac{\delta}{\delta A_{k}} Q_{j k}(A)\right],
$$

where

$$
Q_{j k}(A)=\left\langle\left\{A_{j}, A_{k}\right\}_{\mathrm{PB}} ; A\right\rangle \quad \text { or } \quad\left\langle\left[A_{j}, A_{k}\right] /(i \hbar) ; A\right\rangle .
$$

The important point is that the Poisson-bracket relations depend on the microscopic expressions of the variables. This fact leads to an important consequence that the dynamic universality class of the QCD $C P$ may not be the

\footnotetext{
${ }^{3}$ The model $H[20,31]$ is the minimal-dynamic model for a $C P$ that its relevant modes are given as the nonrelativistichydrodynamic modes. The liquid-gas $C P$ belongs to the dynamic universality class of the model $H$.
} 
same as of the liquid-gas $C P$ or the model $H$. Actually, in the model $H$, the Poisson-bracket relations are calculated with the nonrelativistic relations [20,22].

\section{THE NONLINEAR LANGEVIN EQUATION FOR THE QCD CP}

\section{A. Slow variables}

We first identify the slow variables near the QCD $C P$, which consist of soft modes and conserved densities. For the QCD $C P$, the soft modes are nothing but the longwavelength fluctuations of the conserved densities, i.e., the baryon-number density $n$ and the energy and momentum $T_{\mu \nu}[11,12]$. Thus, we see that the slow variables near the QCD $C P$ are given by only the fluctuations of the conserved quantities:

$$
A_{j}=\left\{\delta n, \delta e=\left(\delta T^{00}\right), \delta J^{i}=\left(\delta T^{0 i}\right)\right\} .
$$

Because the slow dynamics of the conserved quantities is basically given by hydrodynamics, we find that the system near the QCD CP is described as a relativistic critical fluid. In other words, the relevant modes are given as the relativistic hydrodynamic modes. This is the basic observation for our construction of the nonlinear Langevin equation for the QCD CP. More specifically, the hydrodynamic modes are the thermal and viscous diffusion modes, and the sound propagating mode. The thermal mode is the entropy fluctuation inducing the density and energy fluctuations, whereas the viscous and the sound modes are the transverse and longitudinal components of the momentum fluctuations, respectively.

Now, we note that not all fluctuations are enhanced near the $C P$. Therefore, we can neglect nonlinearity of fluctuations that is not enhanced, if such fluctuations are identified. Then, let us identify the nonenhanced fluctuations by the hydrodynamics. The usual hydrodynamics with static scaling laws is useful to see the such tendency of the slow variables. Since the result turns out to be independent of the choice of the frame, which defines the local rest frame [24], let us take the energy frame, namely, the Landau equation [26], which is given by the following conservation laws:

$$
\begin{gathered}
\partial_{\mu} N^{\mu}=0, \\
\partial_{\mu} T^{\mu \nu}=0,
\end{gathered}
$$

where $N^{\nu}$ and $T^{\mu \nu}$ are the particle current and the energymomentum tensor, respectively. Those are given as

$$
\begin{gathered}
N^{\mu}=n u^{\mu}+\nu^{\mu}, \\
T^{\mu \nu}=h u^{\mu} u^{\nu}-P g^{\mu \nu}+\tau^{\mu \nu},
\end{gathered}
$$

where $h=e+P$ is the enthalpy density, with $e$ and $P$ being the energy density and the pressure. Also, $u^{\mu}=$ $(\gamma, \gamma \boldsymbol{v})$ are the fluid four velocity, with $\gamma$ being the
Lorentz factor, and the dissipative terms, $\nu^{\mu}$ and $\tau^{\mu \nu}$, are given by

$$
\begin{gathered}
\nu^{\mu}=\lambda_{0}\left(\frac{n T}{h}\right)^{2} \partial_{\perp}^{\mu}\left(\frac{\mu}{T}\right), \\
\tau^{\mu \nu}=\eta_{0}\left[\partial_{\perp}^{\mu} u^{\nu}+\partial_{\perp}^{\nu} u^{\mu}-\frac{2}{3} \Delta^{\mu \nu}\left(\partial_{\perp} \cdot u\right)\right] \\
+\zeta_{0} \Delta^{\mu \nu}\left(\partial_{\perp} \cdot u\right),
\end{gathered}
$$

where $\lambda_{0}, \eta_{0}$ and $\zeta_{0}$ are the bare thermal conductivity, the bare share and bulk viscosities, respectively. $\Delta^{\mu \nu} \equiv g^{\mu \nu}-$ $u^{\mu} u^{\nu}$ is the projection onto the spacelike vector and $\partial_{\perp}^{\mu} \equiv$ $\Delta^{\mu \nu} \partial_{\nu}$ is the spacelike derivative.

In the hydrodynamic regime, $k \xi \ll 1$, the hydrodynamic mode is analyzed by the linearized equation, which is given by

$$
\begin{gathered}
\frac{\partial \delta n}{\partial t}=-n_{c} \nabla \cdot \delta \boldsymbol{v}+\lambda_{0}\left(\frac{n_{c} T_{c}}{h_{c}}\right)^{2} \nabla^{2} \delta\left(\frac{\mu}{T}\right) \\
\frac{\partial \delta e}{\partial t}=-h_{c} \nabla \cdot \delta \boldsymbol{v} \\
\frac{\partial \delta \boldsymbol{J}}{\partial t}=-\nabla(\delta P)+\left(\zeta_{0}+\frac{1}{3} \eta_{0}\right) \nabla(\nabla \cdot \delta \boldsymbol{v})+\eta_{0} \nabla^{2} \delta \boldsymbol{v},
\end{gathered}
$$

where the symbols with a prefix $\delta$ denote the fluctuations from their equilibrium values, which are denoted by a suffix $c .{ }^{4}$ Hereafter, variables with the suffix and the prefix, respectively, denote the equilibrium values and fluctuations. As relativistic effects, we see that dissipative effects appear in Eq. (20) while vanish in Eq. (21), because we have chosen the energy frame. We note that the relativistic effect for the particle frame appears in a different form [24].

By Eqs. (20)-(23) and static scaling laws, the tendency of the hydrodynamic modes is analyzed in the critical regime, $k \xi \gg 1$. We have studied the such behavior in the previous paper [24] and shown that the thermal mode is enhanced, whereas the sound mode is suppressed and the viscous mode is not enhanced nor suppressed. Recalling the relation between the hydrodynamic modes and the slow variables, we have the result that $\delta n$ and $\delta e$ are enhanced, while $\delta \boldsymbol{J}$ is not near the QCD $C P$.

We note that nonlinear couplings among these modes, which is not included in usual hydrodynamics, become significant in the critical regime. We will take them into account in the nonlinear Langevin equation, except for the fluctuation of the momenta $\delta \boldsymbol{J}$, the nonlinear term of which will be neglected even in the critical regime.

\footnotetext{
${ }^{4}$ Here, we have slightly rewritten the form of the linearized Eqs. (20)-(23), from those in [24] by the thermodynamic relations $\delta \epsilon=T \delta(n s)+\mu \delta n$ and $\delta P=n s \delta T+n \delta \mu$, where $s$ is the entropy per particle.
} 


\section{B. Thermodynamic potential for the slow variables}

Next, we construct the thermodynamic potential $H(\delta n, \delta e, \delta \boldsymbol{J})$ for the slow variables.

Since the momentum density fluctuation is not enhanced near the QCD $C P$, we can neglect its coupling with $\delta n$ and $\delta e$, and may adopt a Gaussian form for the momentum density part of the potential. Thus, we have $H(\delta n, \delta e, J)=$ $H_{n e}(\delta n, \delta e)+H_{J}(\delta \boldsymbol{J})$, with

$$
H_{J}(\delta \boldsymbol{J}) \equiv \frac{1}{2 h_{c}} \delta \boldsymbol{J}^{2} .
$$

In contrast to $\delta \boldsymbol{J}, \delta n$ and $\delta e$ are significantly enhanced near the QCD $C P$; the thermodynamic potential $H_{n e}(\delta n, \delta e)$ should contain higher order terms of them. In fact, $H_{n e}(\delta n, \delta e)$ is the quantity that determines the static property of the system and the QCD $C P$ belongs to the same static universality class as the $3 \mathrm{D}$ Ising class, namely, $Z_{2}$. Therefore, we may construct $H_{n e}(\delta n, \delta e)$ with the thermodynamic potential for the 3D Ising system [32], which reads

$$
\begin{aligned}
\beta H_{\text {Ising }}(\psi, m)= & \int d \boldsymbol{r}\left[\frac{1}{2} r_{0} \psi^{2}+\frac{1}{2} K_{0}|\nabla \psi|^{2}+\frac{1}{4} u_{0} \psi^{4}\right. \\
& \left.+\gamma_{0} \psi^{2} m+\frac{1}{2 C_{0}} m^{2}-h \psi-\tau m\right] .
\end{aligned}
$$

Here, $\psi$ and $m$ are the spin density and the exchange energy density, respectively. $r_{0}, K_{0}, u_{0}, \gamma_{0}$ and $C_{0}$ denote the static parameters, while $h$ and $\tau$ the applied magnetic field and the reduced temperature, respectively. Then, we assume that the thermodynamic potential takes the following form:

$$
H(\delta n, \delta e, \delta \boldsymbol{J})=H_{\text {Ising }}(\psi, m)+\frac{1}{2 h_{c}} \delta \boldsymbol{J}^{2},
$$

provided that the mapping between $(\psi, m)$ and $(\delta n, \delta e)$ is given.

The general mapping relation between a grand canonical ensemble in $Z_{2}$ and the 3D Ising system is known in condensed matter physics [21], which are summarized as follows. First, we assume the following linear relation between the deviations of the respective intensive variables from those at the critical points, which should be valid near the $C P:{ }^{5}$

$$
\begin{aligned}
& \delta h=\alpha_{1} \delta(\mu / T)+\alpha_{2} \delta T / T_{c}, \\
& \delta \tau=\beta_{1} \delta(\mu / T)+\beta_{2} \delta T / T_{c},
\end{aligned}
$$

where $\alpha_{1}, \alpha_{2}, \beta_{1}$ and $\beta_{2}$ are constants and assumed to be regular at the $C P$. We note that $\alpha_{1}, \alpha_{2}, \beta_{1}$ and $\beta_{2}$ need not to be determined for the critical divergence of transport

\footnotetext{
${ }^{5}$ Recall that the static scaling laws are expressed by the deviations of the intensive variables from those at the $C P$.
}

coefficients, since they have no singularities at the $C P$. Although one could use Eqs. (26) and (27) for the mapping, it turns out to be inconvenient for the analysis by a Langevin equation. To translate these equations to more convenient ones, we assume the following relation [21]:

$$
\psi \delta h+m \delta \tau=T_{c}^{-2} \delta T \delta e+\delta(\mu / T) \delta n,
$$

which is actually derived by considering a change of the microscopic distributions due to small deviations of the external parameters in both systems. From the relations Eqs. (26)-(28), we arrive at the convenient mapping relation as follows:

$$
\begin{gathered}
\delta n=\alpha_{1} \psi+\beta_{1} m, \\
T_{c}^{-1} \delta e=\alpha_{2} \psi+\beta_{2} m .
\end{gathered}
$$

With this mapping, Eq. (25) now gives the thermodynamic potential for the QCD CP. We remark that we only map the static quantities although the dynamic ones are studied. For later uses, we introduce fluctuations of the intensive variables as

$$
\begin{gathered}
\delta T \equiv T_{c}^{2} \frac{\delta(\beta H)}{\delta e}, \\
\delta\left(\frac{\mu}{T}\right) \equiv \frac{\delta(\beta H)}{\delta n} .
\end{gathered}
$$

This relation comes from the fact that, in the grand canonical distribution $P_{\text {gra }} \propto \exp [(1 / T) e+(\mu / T) n], e$ and $n$ are, respectively, conjugate to $1 / T$ and $\mu / T$ [21]. We also introduce the fluid velocity fluctuation as in the nonrelativistic case:

$$
\delta \boldsymbol{v} \equiv \frac{\delta H}{\delta \boldsymbol{J}} .
$$

We note that the static parameters in Eq. (24) have the ultraviolet cutoff dependence in the region $\xi^{-1}<\Lambda$. Let us write the static parameters as $r(\Lambda), K(\Lambda), u(\Lambda), \gamma(\Lambda)$, and $C(\Lambda)$ to make their $\Lambda$ dependence explicit. These variables have the following asymptotic behaviors $[21,25,32]$ :

$$
\begin{gathered}
r(\Lambda) \sim \Lambda^{2-\eta}, \\
K(\Lambda) \sim \Lambda^{-\eta}, \\
u(\Lambda) \sim \Lambda^{\epsilon-2 \eta}, \\
\gamma(\Lambda) \sim \Lambda^{(\epsilon+\alpha / \nu) / 2-\eta}, \\
C(\Lambda) \sim \Lambda^{-\alpha / \nu},
\end{gathered}
$$

where $\epsilon=4-d$ with $d$ being the space dimension, while $\alpha, \nu$, and $\eta$ are the usual static critical exponents. Noting that $\eta$ is of order $\epsilon^{2}$ and very small, we neglect $\eta$ and set $K_{0}=1$, hereafter. 


\section{Streaming terms and bare kinetic coefficients}

In this subsection, we determine the forms of the streaming terms, $v_{n}, v_{e}$ and $\boldsymbol{v}_{J}$. We can nicely determine the first two terms from the continuity equations because $\delta n$ and $\delta e$ are the conserved densities. From the continuity equations, we can write $v_{n}$ and $v_{e}$ as divergences of reversible currents, which read

$$
\begin{gathered}
\boldsymbol{j}_{n}=n \gamma \delta \boldsymbol{v}, \\
\boldsymbol{j}_{e}=(e+P) \gamma^{2} \delta \boldsymbol{v},
\end{gathered}
$$

with $\boldsymbol{j}_{n}$ and $\boldsymbol{j}_{e}$ being the reversible currents of the number and energy density, respectively. Here, $\gamma$ is the Lorentz factor of the fluid-velocity fluctuation, $n=n_{c}+\delta n$ and $e=e_{c}+\delta e$. As the reference frame, we have chosen the rest frame of the equilibrium state, and then the background fluid velocity vanishes. Furthermore, We may set $\gamma \sim 1$, because the fluid velocity fluctuation is given by $\delta \boldsymbol{v}=h_{c}^{-1} \delta \boldsymbol{J}$ that is not enhanced. Therefore, we write the streaming terms, $v_{n}$ and $v_{e}$, as

$$
\begin{gathered}
v_{n}=-\nabla \cdot(n \delta \boldsymbol{v}), \\
v_{e}=-\nabla \cdot\left(\left(e+P_{c}\right) \delta \boldsymbol{v}\right),
\end{gathered}
$$

where we neglect the pressure fluctuation because it is not enhanced near the $C P$ [24].

Now, we note that the determination of $\boldsymbol{v}_{J}$ is not simple. Although the continuity equation tells us that $\boldsymbol{v}_{J}$ is the divergence of the reversible-stress tensor, the determination of the reversible-stress tensor is not trivial. However, we can determine it from the potential condition, which is a general condition for the streaming terms [21]. The potential (or divergence) condition $[21,22]$ reads

$$
\int d \boldsymbol{r} \sum_{j=n, e, J} v_{j}(A) \frac{\delta(\beta H)}{\delta A_{j}}=\int d \boldsymbol{r} \sum_{j=n, e, J} \frac{\partial v_{j}(A)}{\partial A_{j}} .
$$

We remark that this condition can be derived from the definition of streaming terms [19]:

$$
v_{j}(A(t)) \equiv\left\langle\dot{A}_{j} ; A(t)\right\rangle,
$$

where $\dot{A}_{j} \equiv i \mathcal{L} A_{j}(0)$ is the microscopic time derivative of $A_{j}$ and $i \mathcal{L}$ is the Liouville operator. In a continuum system, the right-hand side of Eq. (43) vanishes in general [21]. Thus, the potential condition is reduced to

$$
\int d \boldsymbol{r} \sum_{j=n, e, J} v_{j}(A) \frac{\delta(\beta H)}{\delta A_{j}}=0,
$$

where $\boldsymbol{v}_{J}$ is only the unknown quantity because we have already determined $v_{n}, v_{e}$ and $H(\delta n, \delta e, \delta J)$. Using Eqs. (33), (41), (42), and (45), we obtain

$$
\int d \boldsymbol{r}\left[n \nabla \frac{\delta H}{\delta n}+\left(e+P_{c}\right) \nabla \frac{\delta H}{\delta e}+\boldsymbol{v}_{J}\right] \cdot \beta \delta \boldsymbol{v}=0 .
$$

Since this condition should be satisfied for an arbitrary fluid-velocity fluctuation, we have

$$
\boldsymbol{v}_{J}=-n \nabla \frac{\delta H}{\delta n}-\left(e+P_{c}\right) \nabla \frac{\delta H}{\delta e} .
$$

Next, let us determine the kinetic coefficients from the relativistic hydrodynamic equation, Eqs. (14)-(19). From Eqs. (18), (19), (32), and (33), we can read the kinetic coefficients $L_{j k}$ for small $\delta \boldsymbol{v}$ as

$$
L_{n n}=-\lambda_{0}\left(\frac{n_{c} T_{c}}{h_{c}}\right)^{2} \nabla^{2},
$$

$$
L_{J J}^{i j}=-T_{c}\left[\eta_{0} \delta_{i j} \partial_{i} \partial_{j}+\left(\zeta_{0}+(1-2 / d) \eta_{0}\right) \partial_{i} \partial_{j}\right],
$$

and that the other coefficients are zero. We note that $L_{e e}$ is absent due to the choice of the energy frame.

Now, we have determined all the necessary terms, and then can write down the nonlinear Langevin equation for the QCD $C P$ as

$$
\begin{gathered}
\frac{\partial \delta n}{\partial t}=-\nabla \cdot(n \delta \boldsymbol{v})-L_{n n} \frac{\delta(\beta H)}{\delta n}+\theta_{n}, \\
\frac{\partial \delta e}{\partial t}=-\nabla \cdot\left(\left(e+P_{c}\right) \delta \boldsymbol{v}\right), \\
\frac{\partial \delta \boldsymbol{J}}{\partial t}=-n \nabla \frac{\delta H}{\delta n}-\left(e+P_{c}\right) \nabla \frac{\delta H}{\delta e}-L_{J J} \cdot \frac{\delta(\beta H)}{\delta \boldsymbol{J}}+\boldsymbol{\theta}_{J},
\end{gathered}
$$

where $\theta_{n}$ and $\boldsymbol{\theta}_{J}$ are the noise terms and satisfy the fluctuation-dissipation relations

$$
\left\langle\theta_{n}(\boldsymbol{r}, t) \theta_{n}\left(\boldsymbol{r}^{\prime}, t^{\prime}\right)\right\rangle=-2 \lambda_{0}\left(\frac{n_{c} T_{c}}{h_{c}}\right)^{2} \nabla^{2} \delta\left(\boldsymbol{r}-\boldsymbol{r}^{\prime}\right) \times \delta\left(t-t^{\prime}\right),
$$

$$
\begin{aligned}
\left\langle\theta_{J}^{i}(\boldsymbol{r}, t)\right. & \left.\theta_{J}^{i}\left(\boldsymbol{r}^{\prime}, t^{\prime}\right)\right\rangle \\
= & -2 T_{c}\left[\eta_{0} \delta^{i j} \nabla^{2}+\left\{\zeta_{0}+(1-2 / d) \eta_{0}\right\} \partial^{i} \partial^{j}\right] \\
\quad & \times \delta\left(\boldsymbol{r}-\boldsymbol{r}^{\prime}\right) \delta\left(t-t^{\prime}\right) .
\end{aligned}
$$

Let us write the transport coefficients as $\lambda(\Lambda), \eta(\Lambda)$ and $\zeta(\Lambda)$ to make their cutoff dependence in the region, $\xi^{-1}<\Lambda$. The critical behaviors of the transport coefficients are determined from their asymptotic behaviors near the relevant-fixed point as $\Lambda$ is lowered.

Here, we compare the Langevin equation Eqs. (50)-(52) with that for the liquid-gas $C P$ [25]

$$
\frac{\partial \delta n}{\partial t}=-\nabla \cdot(n \delta v)
$$

$$
\frac{\partial \delta e}{\partial t}=-\nabla \cdot\left(\left(e+P_{c}\right) \delta v\right)+\lambda_{0} T_{c} \nabla^{2} \frac{\delta H^{\mathrm{lg}}}{\delta e}+\theta_{e},
$$




$$
\begin{aligned}
\frac{\partial \delta \boldsymbol{J}_{\rho}}{\partial t}= & -n \nabla \frac{\delta H^{\mathrm{lg}}}{\delta n}-\left(e+P_{c}\right) \nabla \frac{\delta H^{\mathrm{lg}}}{\delta e} \\
& -L_{J J} \cdot \frac{\delta\left(\beta H^{\mathrm{lg}}\right)}{\delta \boldsymbol{J}_{\rho}}+\boldsymbol{\theta}_{J},
\end{aligned}
$$

where $\delta \boldsymbol{J}_{\rho} \equiv \rho_{c} \delta \boldsymbol{v}, \rho$ and $H^{\mathrm{lg}}$ are the nonrelativistic momentum density, the mass density and the thermodynamic potential for liquid-gas $C P$, respectively:

$$
H^{\lg }(\delta n, \delta e, \delta \boldsymbol{J})=H_{\text {Ising }}(\psi, m)+\frac{1}{2 \rho_{c}} \delta \boldsymbol{J}^{2} .
$$

We see that the streaming terms have the same forms but the dissipative ones are totally different between the relativistic and nonrelativistic cases. The difference also appears in the relation between the momentum and the fluid velocity fluctuation. Therefore, one may naturally expect some novel characteristics in the relativistic case that are absent in the nonrelativistic case [25].

\section{THE TRANSPORT COEFFICIENTS BY DYNAMIC RG}

We here present an analysis of transport coefficients by the dynamic RG. A detailed derivation of the RG equations is given in the Appendixes.

First, we rewrite Eqs. (50)-(52) as the equation for $\psi$ and $m$ to conform the hydrodynamic variables, $\delta n$ and $\delta e$, to the Ising variables, $\psi$ and $m$. Noting that we can set $\alpha_{2}=0$ in the mapping relations, Eqs. (29) and (30), without loss of generality [25], we have

$$
\begin{gathered}
\frac{\partial \psi}{\partial t}=-C_{\psi} \nabla \cdot \delta \boldsymbol{J}-\alpha_{1}^{-2} L_{n n} \frac{\delta(\beta H)}{\delta \psi} \\
-h_{c}^{-1} \nabla \cdot(\psi \delta \boldsymbol{J})+\alpha_{1}^{-1} \theta_{n}, \\
\frac{\partial m}{\partial t}=-\beta_{2}^{-1} \nabla \cdot \delta \boldsymbol{J}-h_{c}^{-1} \nabla \cdot(m \delta \boldsymbol{J}), \\
\frac{\partial(\delta \boldsymbol{J})}{\partial t}=-C_{J} \nabla \frac{\delta H}{\delta \psi}-\beta_{2}^{-1} h_{c} \nabla \frac{\delta H}{\delta m}-\psi \nabla \frac{\delta H}{\delta \psi} \\
-m \nabla \frac{\delta H}{\delta m}-\left(T_{c} h_{c}\right)^{-1} L_{J J} \cdot \delta \boldsymbol{J}+\boldsymbol{\theta}_{J},
\end{gathered}
$$

with $C_{\psi} \equiv \alpha_{1}^{-1}\left(n_{c} h_{c}^{-1}-\beta_{1} \beta_{2}^{-1}\right)$ and $C_{J} \equiv \alpha_{1}^{-1}\left(n_{c}-\right.$ $\left.\beta_{1} h_{c}\right)$. Here, we note that we could rewrite the potential, Eq. (24), for $\psi$ and $m$ as that for $\delta n$ and $\delta e$ to conform the variables; the choice is a matter of preference.

In the dynamic RG transformation, we average over the short-wavelength components in the shell, $\Lambda-\delta \Lambda<$ $k<\Lambda$, for the Langevin equation. For this task, we must perturbatively solve the equation about them, by rewriting it as a self-consistent equation [22]. Although an explicit derivation of the self-consistent equation for the QCD $C P$ is first made in this paper, we leave the details of the derivation to Appendix A, because the general procedure of the derivation is standard and given in the textbook [22]. Here, we present only a few basic equations of the dynamic $\mathrm{RG}$ for the QCD CP. Now, as is shown in Appendix A, Eqs. (59)-(61) can be reduced to the following form:

$$
\left(\begin{array}{c}
\tilde{\psi}(\boldsymbol{k}, \omega) \\
\tilde{m}(\boldsymbol{k}, \omega) \\
\delta \tilde{J}_{\|}(\boldsymbol{k}, \omega)
\end{array}\right)=\left(\begin{array}{c}
\tilde{\psi}^{0}(\boldsymbol{k}, \omega) \\
0 \\
\delta \tilde{J}_{\|}^{0}(\boldsymbol{k}, \omega)
\end{array}\right)+G^{0}(\boldsymbol{k}, \omega) \boldsymbol{V}(\boldsymbol{k}, \omega),
$$

and

$$
\delta \tilde{\boldsymbol{J}}_{\perp}(\boldsymbol{k}, \omega)=\delta \tilde{\boldsymbol{J}}_{\perp}^{0}(\boldsymbol{k}, \omega)+G_{\perp}^{0} \boldsymbol{V}_{\perp \psi \psi}(\boldsymbol{k}, \omega),
$$

where $\tilde{J}_{\|}(\boldsymbol{k}) \equiv \hat{\boldsymbol{k}} \cdot \tilde{J}(\boldsymbol{k})$ and $\tilde{J}_{\perp}(\boldsymbol{k}) \equiv \tilde{J}(\boldsymbol{k})-\tilde{J}_{\|}(\boldsymbol{k})$ are the longitudinal and transverse components of the momentum. Here, $G^{0}$ and $G_{\perp}^{0}$ are the bare propagators, which are given by Eqs. (A50)-(A53), whereas $\boldsymbol{V}$ and $\boldsymbol{V}_{\perp \psi \psi}$ the nonlinear couplings, coming from the streaming terms and given by Eqs. (A29)-(A34) and (A59). Also, $\tilde{\psi}^{0}, \delta \tilde{J}_{\|}^{0}$ and $\delta \tilde{\boldsymbol{J}}_{\perp}^{0}$ are the bare variables, which are the solutions without the nonlinear terms. Iterating the self-consistent Eqs. (62) and (63), we can obtain a perturbative expansion of the nonlinear couplings and have a coarse-grained Langevin equation.

Now, we note that the variables, $\psi, \tilde{J}_{\perp}$, and $\tilde{J}_{\|}$correspond, respectively, to the thermal, viscous, and sound modes $^{6}$ (see the propagators (A51)-(A53). Therefore, the first and third rows of Eq. (62) respectively denote the equations of motion for the thermal and sound modes, while Eq. (63) for the viscous mode. We stress that the sound mode is neglected in the model $H$, although it is essential for the renormalization of the bulk viscosity.

Here, we make the coarse-graining to the second order in the nonlinear couplings, $\boldsymbol{V}$ and $\boldsymbol{V}_{\perp \psi \psi}$ (see Fig. 3 for an example.). Inspecting the coarse-grained equation for $\tilde{\psi}$ (see Eq. (B5) for the detail) and, we have the RG equation for the thermal conductivity:

$$
-\Lambda \frac{\partial \lambda(\Lambda)}{\partial \Lambda}=\frac{3}{4} f(\Lambda) \lambda(\Lambda),
$$

$f(\Lambda) \equiv T_{c} K_{4} /\left(D_{\psi} \eta(\Lambda) \lambda(\Lambda) \Lambda^{\epsilon}\right), K_{4}$ is the surface area of a unit sphere in four dimensions divided by $(2 \pi)^{4}, D_{\psi} \equiv$ $\left(n_{c} T_{c} / \alpha_{1} h_{c}\right)^{2}$. Here, we have introduced $f(\Lambda)$ for convenience sake. Similarly, from the coarse-grained equations for $\tilde{J}_{\perp}$ and $\tilde{J}_{\|}$, we obtain the RG equations for the shear and bulk viscosities

$$
\begin{gathered}
-\Lambda \frac{\partial \eta(\Lambda)}{\partial \Lambda}=\frac{1}{24} f(\Lambda) \eta(\Lambda), \\
-\Lambda \frac{\partial \zeta(\Lambda)}{\partial \Lambda}=A \gamma^{2}(\Lambda) \lambda^{-1}(\Lambda) \Lambda^{-\epsilon-4},
\end{gathered}
$$

\footnotetext{
${ }^{6}$ Although $\tilde{m}$ would be a linear combination of the thermal and sound modes, we need not to consider $\tilde{m}$ for a following analysis.
} 
where $\gamma(\Lambda)$ is a static parameter in the thermodynamic potential (see Eqs. (24) and (37)), and $A \equiv h_{c}^{2} K_{4} /\left(\beta_{2}^{2} D_{\psi}\right)$. Furthermore, differentiating $f(\Lambda)$ about $\Lambda$, we also have the $\mathrm{RG}$ equation for it:

$$
-\Lambda \frac{\partial f(\Lambda)}{\partial \Lambda}=f(\Lambda)\left(\epsilon-\frac{19}{24} f(\Lambda)\right) .
$$

Now, we note that Eqs. (64), (65), and (67) are identical to those for the liquid-gas $C P$ except for unimportant constants in $f(\Lambda)[21,25]$. Equation (66) is also equivalent to the RG equation of the bulk viscosity for the liquid-gas $C P$ in the limit $\omega \rightarrow 0[21,25]$. Therefore, arguments about the RG equations and results from those are the same as for the liquid-gas $C P$. Then, we provide only essential arguments and results in the following part, and leave the detail to $[21,25,28,29]$.

Now, we identify the relevant fixed point as the following $[21,25]$. Because, at a fixed point, parameters are invariant about the RG transformation, we set the lefthand side of Eq. (67) as 0. Then, as a fixed-point value of $f(\Lambda)$ which is denoted by $f^{*}$, we have $f^{*}=0$ and $f^{*}=$ $(24 / 19) \epsilon$. Therefore, we have the two fixed points and the relevant one is specified by $f^{*}=(24 / 19) \epsilon$. Although the relevant point seems to be absent in Eqs. (64)-(66), the reason is due to the simplified RG transformation as mentioned in the earlier section, and this is just an apparent problem $[28,29]$.

Substituting $f^{*}=(24 / 19) \epsilon$ into Eqs. (64)-(66), we have the asymptotic behaviors near the relevant-fixed point:

$$
\begin{gathered}
\lambda(\Lambda) \sim \Lambda^{-(18 / 19) \epsilon}, \\
\eta(\Lambda) \sim \Lambda^{-(1 / 19) \epsilon}, \\
\zeta(\Lambda) \sim \Lambda^{-(4-(18 / 19) \epsilon-(\alpha / \nu))} .
\end{gathered}
$$

Here, in the derivation of Eq. (70), we have used the asymptotic behavior of $\gamma(\Lambda)$, Eq. (37). Decreasing the cutoff to the region $\Lambda \ll \xi^{-1}$, we can replace $\Lambda$ with $\xi^{-1}$ in the asymptotic behaviors [21,28]:

$$
\begin{gathered}
\lambda_{R} \sim \xi^{(18 / 19) \epsilon,} \\
\eta_{R} \sim \xi^{(1 / 19) \epsilon,} \\
\zeta_{R} \sim \xi^{4-(18 / 19) \epsilon-(\alpha / \nu) .}
\end{gathered}
$$

In three dimensions, we find

$$
\begin{gathered}
\lambda_{R} \sim \xi^{0.95}, \\
\eta_{R} \sim \xi^{0.053}, \\
\zeta_{R} \sim \xi^{2.8} .
\end{gathered}
$$

We can also read the dynamic critical exponents from Eqs. (71)-(73). A dynamic critical exponent, denoted by $z$, generically parametrizes the decay rate $\Gamma(k)$ at the wave number $k=\xi^{-1}$ as $\Gamma\left(\xi^{-1}\right) \sim \xi^{-z}$. As shown in Appendix A, the decay rates for the three modes at $k$ are given by

$$
\begin{gathered}
\Gamma_{\text {thermal }}(k)=\lambda_{R} k^{2}\left(r_{R}+k^{2}\right) D_{\psi}, \\
\Gamma_{\text {viscous }}(k)=\eta_{R} k^{2} h_{c}^{-1}, \\
\Gamma_{\text {sound }}(k)=\left(\zeta_{R}+2(1-1 / d) \eta_{R}\right) k^{2} h_{c}^{-1} .
\end{gathered}
$$

Thus, we find the dynamic critical exponents as

$$
\begin{gathered}
z_{\text {thermal }}=4-\frac{18}{19} \epsilon, \\
z_{\text {viscous }}=2-\frac{1}{19} \epsilon, \\
z_{\text {sound }}=-\left(2-\frac{18}{19} \epsilon-\frac{\alpha}{\nu}\right) .
\end{gathered}
$$

In three dimensions, the dynamic critical exponents are given by

$$
\begin{gathered}
z_{\text {thermal }} \sim 3, \\
z_{\text {viscous }} \sim 2, \\
z_{\text {sound }} \sim-0.8 .
\end{gathered}
$$

We see that the thermal and viscous modes exhibit critical slowing down, while the sound mode critical speeding up.

Why do the relativistic effects not appear in the RG equations? The reason is that the nonlinear terms in the dissipative terms generally renormalize only static parameters, up to order $\epsilon^{2}$ [22,28]. Furthermore, the difference in the relation between the momentum and the fluid velocity is only unimportant constants, i.e., the enthalpy density $h$ and the mass density $\rho$. Then, the RG equations are essentially the same as for the nonrelativistic case.

\section{SUMMARY AND CONCLUDING REMARKS}

We have studied the critical behaviors of the transport coefficients and the dynamic critical exponents at the QCD critical point $(C P)$ by dynamic renormalization group (RG). For this purpose, we have constructed the nonlinear Langevin equation near the QCD $C P$ for the first time. Our construction is based on the generalized Langevin theory, by Mori $[19,23]$, and the relativistic hydrodynamics; instead of a naive construction method [22], we have determined the streaming terms by the relativistic hydrodynamics and the potential condition that gives a constraint to these terms. The resulting equation is given by Eqs. (50)-(52). Although there are some attempts to make a one-to-one mapping between $\mathrm{QCD} C P$ and Ising $C P$ 
$[17,33]$, we have shown that it is not necessary to specify such the mapping for the critical exponents, as for the liquid-gas $C P$ [21].

We have shown that the bulk viscosity and the thermal conductivity strongly diverge at the QCD $C P$. Also, we have found that the thermal and viscous diffusion modes exhibit critical slowing down with the dynamic critical exponents $z_{\text {thermal }} \sim 3$ and $z_{\text {viscous }} \sim 2$, respectively. In contrast, the sound propagating mode critical speeding up with the negative exponent $z_{\text {sound }} \sim-0.8$.

We now compare our result about the bulk viscosity to that in [15]. Although a divergent behavior of the bulk viscosity is the same, the critical exponent is different in the two cases. In [15], the critical exponent is estimated to be about 0.2 and the divergence is weak contrary to our result. We also note that the study by the relativistic Boltzmann equation [18] gives only the bare bulk viscosity.

We note that the bulk viscosity and the thermal conductivity are usually neglected in heavy ion physics, however they become much more important than the shear viscosity near the QCD $C P$. Furthermore, the description for the created matter as a perfect fluid is not valid near the QCD $C P$ at all due to the strong divergence of the bulk viscosity.

As the argument about the dynamic universality class $[12,17]$, we have shown, from an explicit calculation, that the QCD $C P$ has the same critical behaviors as the liquidgas $C P$ has. The argument assumes the insignificance of the relativity for the critical dynamics by the slowness of the diffusion processes. However, we have shown that the genuine reason for the insignificance originates from the small fluctuation of the momentum density; the critical dynamics is essentially governed by the streaming terms, which are modified by the relativistic effect through only a Lorentz factor of the fluid-velocity fluctuation. However, the fluid-velocity fluctuation, which is proportional to the momentum, is not enhanced near the $C P$. Thus, the relativistic effects do not affect the critical dynamics near the QCD $C P$. We stress that the sound mode exhibit critical speeding up, and then the sound diffusion is fast near the QCD $C P$. Therefore, the basis of the conjecture would be true for the thermal and viscous modes, but not for the sound mode. We also note that the model $H$ [20], which is the minimaldynamic model for the dynamics near the liquid-gas $C P$, can not describe the critical behavior of the bulk viscosity because it does not contain the sound mode.

We note that our Langevin equation must satisfy usual fluctuation-dissipation relations, Eqs. (53) and (54), for the consistency with the linearized Landau equation, ${ }^{7}$ although a relativistic Brownian motion seems not to satisfy the usual relations [34]. Moreover, our Langevin equation seems to violate the causality, since the dissipative terms

\footnotetext{
${ }^{7}$ If our nonlinear Langevin equation is linearized, the linearized equation must give the same result as the Landau equation gives.
}

are determined from the Landau equation. However, the Israel-Stewart equation [35], in which the causality problem is formally resolved, gives the same result as the Landau equation gives in the long-wavelength region [24]. Therefore, our determination from the Landau equation must suffice. Furthermore, we note that short-wavelength components in the region, $k>a^{-1}$ where $a$ is a characteristic microscopic length, would violate the causality. Therefore, we can exclude such the illegal components from the theory by the cutoff, $\Lambda_{0} \ll a^{-1}$. We stress that all infrared effective theories inevitably have the ultraviolet cutoff; naturally, relativistic hydrodynamics also has it.

Also, we note a frame dependence of the results. As a hydrodynamic equation, we used only the equation in the energy frame. Do the results change if an equation in the particle frame is used? Although the frame dependence can appear in only dissipative terms, the critical dynamics is essentially determined by the streaming terms. Therefore, the results would not change for the particle frame, if an equation in the frame is correct. However, in practice, the Eckart equation has a pathological behavior [36]. Namely, fluctuations do not relax, and therefore we cannot use the Eckart equation.

Recently, some authors have suggested the existence of other critical points in higher density region of the QCD phase diagram where the color superconductivity is taken into account $[2,3]$. It would be interesting to study the critical dynamics near such a new QCD $C P$ using the dynamic RG theory, as an extension of the present work. For this purpose, however, we must firstly specify the soft modes and construct the nonlinear Langevin equation. If the soft modes are different from the conserved densities, which is the case when the diquark fluctuations are relevant $[2,37]$, the construction based on the relativistic hydrodynamics done in the present work does not work, and we must directly recourse to Eq. (11) to identify the streaming terms.

\section{ACKNOWLEDGMENTS}

We are grateful to Hideo Suganuma for his useful comments. We also thank Teiji Kunihiro for his careful reading of this paper. This work was supported by the Global COE Program "The Next Generation of Physics, Spun from Universality and Emergence" in Kyoto University, by the Yukawa International Program for Quark-hadron Sciences in YITP and by the Grant-in-Aid for Scientific Research in Japan [Nos. 22-1050].

\section{APPENDIX A: REWRITING THE NONLINEAR LANGEVIN EQUATION AS A SELF-CONSISTENT EQUATION}

Here, we rewrite the Langevin equation, Eqs. (59)-(61) as a self-consistent equation. First, we make a Fourier transformation as the following: 
YUKI MINAMI

$$
\tilde{\psi}(\boldsymbol{k}, \omega)=\int d t d^{d} r e^{i \omega t-i \boldsymbol{k} \cdot \boldsymbol{r}} \psi(\boldsymbol{r}, t) .
$$

Then, we have

$$
\begin{gathered}
-i \omega \tilde{\psi}(\boldsymbol{k}, \omega)=-C_{\psi} i \boldsymbol{k} \cdot \delta \tilde{\boldsymbol{J}}-\alpha_{1}^{-2} \tilde{L}_{n n} \frac{\delta(\beta \tilde{H})}{\delta \psi}-h_{c}^{-1} i \boldsymbol{k} \cdot \int_{q \Omega}(\tilde{\psi}(q) \delta \tilde{\boldsymbol{J}}(k-q))+\alpha_{1}^{-1} \tilde{\theta}_{n}, \\
-i \omega \tilde{m}(\boldsymbol{k}, \omega)=-\beta_{2}^{-1} i \boldsymbol{k} \cdot \delta \tilde{\boldsymbol{J}}-h_{c}^{-1} i \boldsymbol{k} \cdot \int_{q \Omega}(\tilde{m}(q) \delta \tilde{\boldsymbol{J}}(k-q)), \\
-i \omega \delta \tilde{\boldsymbol{J}}(\boldsymbol{k}, \omega)=-C_{J} i \boldsymbol{k} \frac{\delta \tilde{H}}{\delta \psi}-\beta_{2}^{-1} h_{c} i \boldsymbol{k} \frac{\delta \tilde{H}}{\delta m}-i \int_{q \Omega} q\left[\frac{\delta \tilde{H}}{\delta \psi}(q) \tilde{\psi}(k-q)+\frac{\delta \tilde{H}}{\delta m}(q) \tilde{m}(k-q)\right]-\left(T_{c} h_{c}\right)^{-1} \tilde{L}_{J J} \cdot \delta \tilde{\boldsymbol{J}}+\tilde{\boldsymbol{\theta}}_{J} .
\end{gathered}
$$

Note that the quantities with a tilde in Eq. (A2)-(A4) are Fourier transformed, like Eq. (A1), and we have abbreviated the nonlinear terms such as

$$
\int_{q \Omega} \tilde{\psi}(q) \delta \tilde{\boldsymbol{J}}(k-q) \equiv \int \frac{d \Omega}{2 \pi} \frac{d^{d} q}{(2 \pi)^{d}} \tilde{\psi}(\boldsymbol{q}, \Omega) \delta \tilde{\boldsymbol{J}}(\boldsymbol{k}-\boldsymbol{q}, \omega-\Omega) .
$$

We now decompose Eq. (A4) into the longitudinal and the transverse components:

$$
\begin{gathered}
-i \omega \delta \tilde{\boldsymbol{J}}_{\|}=-i C_{J} k \frac{\delta \tilde{H}}{\delta \psi}-i \beta_{2}^{-1} h_{c} k \frac{\delta \tilde{H}}{\delta m}-i \int_{q \Omega}(\hat{\boldsymbol{k}} \cdot \boldsymbol{q})\left[\frac{\delta \tilde{H}}{\delta \psi}(q) \tilde{\psi}(k-q)+\frac{\delta \tilde{H}}{\delta m}(q) \tilde{m}(k-q)\right]-\left(T_{c} H_{c}\right)^{-1} \hat{\boldsymbol{k}} \cdot \tilde{L}_{J J}(\boldsymbol{k}) \cdot \delta \tilde{\boldsymbol{J}}+\tilde{\theta}_{\|}, \\
-i \omega \delta \tilde{\boldsymbol{J}}_{\perp}=-i \int_{q \Omega} \mathcal{P}_{\perp}(\boldsymbol{k}) \cdot \boldsymbol{q}\left[\frac{\delta \tilde{H}}{\delta \psi}(q) \tilde{\psi}(k-q)+\frac{\delta \tilde{H}}{\delta m}(q) \tilde{m}(k-q)\right]-\left(T_{c} h_{c}\right)^{-1} \mathcal{P}_{\perp}(\boldsymbol{k}) \cdot \tilde{L}_{J J}(\boldsymbol{k}) \cdot \delta \tilde{\boldsymbol{J}}+\tilde{\boldsymbol{\theta}}_{\perp},
\end{gathered}
$$

where we have introduced a projection operator as

$$
\left(\mathcal{P}_{\perp}(\boldsymbol{k})\right)_{i j}=\delta_{i j}-k_{i} k_{j} / k^{2},
$$

and

$$
\begin{gathered}
\delta \tilde{J}_{\|}(\boldsymbol{k})=\hat{\boldsymbol{k}} \cdot \delta \tilde{\boldsymbol{J}}(\boldsymbol{k}), \\
\delta \tilde{\boldsymbol{J}}_{\perp}(\boldsymbol{k})=\mathcal{P}_{\perp}(\boldsymbol{k}) \cdot \delta \tilde{\boldsymbol{J}}(\boldsymbol{k}), \\
\tilde{\theta}_{\|}(\boldsymbol{k})=\hat{\boldsymbol{k}} \cdot \tilde{\boldsymbol{\theta}}(\boldsymbol{k}), \\
\tilde{\boldsymbol{\theta}}_{\perp}(\boldsymbol{k})=\mathcal{P}_{\perp}(\boldsymbol{k}) \cdot \tilde{\boldsymbol{\theta}}(\boldsymbol{k}) .
\end{gathered}
$$

Because the streaming terms in Eqs. (A6) and (A7) are too complicated for our purpose, let us retain only the terms that yield dominant contributions for the transport coefficients. We note that only such terms suffice for obtaining the critical exponents. From the relations [21]

$$
\begin{aligned}
& \int d^{3} r\langle\psi(\boldsymbol{r}) \psi(0)\rangle \sim \xi^{2}, \\
& \int d^{3} r\langle m(\boldsymbol{r}) m(0)\rangle \sim \xi^{0.2},
\end{aligned}
$$

we expect $\psi$ yields stronger singularity than $m$. Therefore, we only retain the term that are of the second order in $\psi$. Namely, we reduce the streaming terms as

$$
\begin{gathered}
i C_{J} k \frac{\delta \tilde{H}}{\delta \psi}+i \beta_{2}^{-1} h_{c} k \frac{\delta \tilde{H}}{\delta m}+i \int_{q \Omega}(\hat{\boldsymbol{k}} \cdot \boldsymbol{q})\left[\frac{\delta \tilde{H}}{\delta \psi}(q) \tilde{\psi}(k-q)+\frac{\delta \tilde{H}}{\delta m}(q) \tilde{m}(k-q)\right] \\
\sim T_{c}\left[i C_{J} k \chi_{0}^{-1}(k) \tilde{\psi}+i \beta_{2}^{-1} h_{c} k C_{0}^{-1} \tilde{m}+i \beta_{2}^{-1} h_{c} k \gamma_{0} \int_{q \Omega} \tilde{\psi}(q) \tilde{\psi}(k-q)\right], \\
i \int_{q \Omega} \mathcal{P}_{\perp}(\boldsymbol{k}) \cdot \boldsymbol{q}\left[\frac{\delta \tilde{H}}{\delta \psi}(q) \tilde{\psi}(k-q)+\frac{\delta \tilde{H}}{\delta m}(q) \tilde{m}(k-q)\right] \sim i T_{c} \mathcal{P}_{\perp}(\boldsymbol{k}) \cdot \int_{q \Omega} \boldsymbol{q} \chi_{0}^{-1}(\boldsymbol{q}) \tilde{\psi}(q) \tilde{\psi}(k-q),
\end{gathered}
$$


where $\chi_{0}^{-1}(\boldsymbol{k})=r_{0}+k^{2}$. Notice that we have set $K_{0}=1$, as mentioned in the text.

Next, we consider the dissipative terms. The important point is that the nonlinear terms in dissipative terms renormalize only static parameters in a thermodynamic potential to second order in $\epsilon$, generally [22,28]. Therefore, we can take into account nonlinear terms in the dissipative terms with the results of static RG, Eq. (34)-(38), and effectively neglect it in the Langevin equation. Then, we reduce the $\tilde{L}_{n n} \delta(\beta \tilde{H}) / \delta \psi$ as

$$
\tilde{L}_{n n}(\boldsymbol{k}) \frac{\delta \tilde{H}}{\delta \psi}(\boldsymbol{k}, \omega) \sim \lambda_{0} k^{2} \chi_{0}^{-1}\left(\frac{n_{c} T_{c}}{h_{c}}\right)^{2} \tilde{\psi}(\boldsymbol{k}, \omega) .
$$

In contrast, the dissipative terms of $\delta \boldsymbol{J}$ are originally linear and then directly read

$$
\begin{aligned}
& \hat{\boldsymbol{k}} \cdot \tilde{L}_{J J}(\boldsymbol{k}) \cdot \delta \boldsymbol{J}(\boldsymbol{k}, \omega)= T_{c}\left[\zeta_{0}+2(1-1 / d) \eta_{0}\right] \\
& \times k^{2} \delta \tilde{J}_{\|}(\boldsymbol{k}, \omega), \\
& \mathcal{P}_{\perp}(\boldsymbol{k}) \cdot \tilde{L}_{J J}(\boldsymbol{k}) \cdot \delta \boldsymbol{J}(\boldsymbol{k}, \omega)=T_{c} \eta_{0} k^{2} \delta \tilde{\boldsymbol{J}}_{\perp}(\boldsymbol{k}, \omega) .
\end{aligned}
$$

Collecting the above results, we arrive at the reduced nonlinear Langevin equation as given by

$$
\begin{gathered}
-i \omega \tilde{\psi}=-i k C_{\psi} \delta \tilde{J}_{\|}-h_{c}^{-1} i \boldsymbol{k} \cdot \int_{q \Omega} \tilde{\psi}(q) \delta \tilde{\boldsymbol{J}}(k-q)-\lambda_{0} k^{2} D_{\psi} \chi_{0}^{-1}(\boldsymbol{k}) \tilde{\psi}+\alpha_{1}^{-1} \tilde{\boldsymbol{\theta}}_{n}, \\
-i \omega \tilde{m}=-\beta_{2}^{-1} i k \delta \tilde{J}_{\|}-h_{c}^{-1} i \boldsymbol{k} \cdot \int_{q \Omega} \tilde{m}(q) \delta \tilde{\boldsymbol{J}}(k-q), \\
-i \omega \delta \tilde{J}_{\|}=T_{c}\left[-i k \chi_{0}^{-1}(\boldsymbol{k}) C_{J} \tilde{\psi}-i k C_{0}^{-1} \beta_{2} h_{c} \tilde{m}-i k \beta_{2}^{-1} h_{c} \gamma_{0} \int_{q \Omega} \tilde{\psi}(q) \tilde{\psi}(k-q)\right]-k^{2} \nu_{0}^{l} h_{c}^{-1} \delta \tilde{\boldsymbol{J}}_{\|}+\tilde{\theta}_{\|}, \\
-i \omega \delta \tilde{\boldsymbol{J}}_{\perp}=-i T_{c} \mathcal{P}_{\perp}(\boldsymbol{k}) \cdot \int_{q \Omega} \boldsymbol{q} \chi_{0}^{-1}(\boldsymbol{q}) \tilde{\psi}(q) \tilde{\psi}(k-q)-k^{2} \eta_{0} h_{c}^{-1} \delta \tilde{\boldsymbol{J}}_{\perp}+\tilde{\boldsymbol{\theta}}_{\perp},
\end{gathered}
$$

where

$$
\begin{gathered}
D_{\psi} \equiv\left(\frac{n_{c} T_{c}}{\alpha_{1} h_{c}}\right)^{2}, \\
\nu_{0}^{l} \equiv\left[\zeta_{0}+2(1-1 / d) \eta_{0}\right] .
\end{gathered}
$$

This is the basic equation for the dynamics near the QCD $C P$, which is first written down, and is a main result of this paper.

We can compactly rewrite the basic equation in a matrix form:

$$
\mathcal{M}(\boldsymbol{k}, \omega)\left(\begin{array}{c}
\tilde{\psi}(\boldsymbol{k}, \omega) \\
\tilde{m}(\boldsymbol{k}, \omega) \\
\delta \tilde{J}_{\|}(\boldsymbol{k}, \omega)
\end{array}\right)=\boldsymbol{V}(\boldsymbol{k}, \omega)+\boldsymbol{\theta}(\boldsymbol{k}, \omega)
$$

where

$$
\begin{gathered}
\mathcal{M}(\boldsymbol{k}, \omega)=\left(\begin{array}{ccc}
-i \omega+\lambda_{0} k^{2} D_{\psi} \chi_{0}^{-1}(\boldsymbol{k}) & 0 & i k C_{\psi} \\
0 & -i \omega & i k \beta_{2}^{-1} \\
i k \chi_{0}^{-1}(\boldsymbol{k}) C_{J} T_{c} & i k C_{0}^{-1} \beta_{2} h_{c} T_{c} & -i \omega+k^{2} \nu_{0}^{l} h_{c}^{-1}
\end{array}\right), \\
\boldsymbol{\theta}(\boldsymbol{k}, \omega)=\left(\begin{array}{c}
\alpha_{1}^{-1} \tilde{\theta}(\boldsymbol{k}, \omega) \\
0 \\
\tilde{\theta}_{\|}(\boldsymbol{k}, \omega)
\end{array}\right), \\
\boldsymbol{V}(\boldsymbol{k}, \omega)=\left(\begin{array}{c}
V_{\psi \psi \perp}(\boldsymbol{k}, \omega)+V_{\psi \psi \|}(\boldsymbol{k}, \omega) \\
V_{m m \perp}(\boldsymbol{k}, \omega)+V_{m m \|}(\boldsymbol{k}, \omega) \\
V_{\| \psi \psi}(\boldsymbol{k}, \omega)
\end{array}\right),
\end{gathered}
$$

and

$$
V_{\psi \psi \perp}(\boldsymbol{k}, \omega) \equiv-h_{c}^{-1} i \boldsymbol{k} \cdot \int_{q \Omega} \tilde{\psi}(q) \delta \tilde{\boldsymbol{J}}_{\perp}(k-q),
$$




$$
\begin{gathered}
V_{\psi \psi \|}(\boldsymbol{k}, \omega) \equiv-h_{c}^{-1} i \int_{q \Omega} \boldsymbol{k} \cdot(\boldsymbol{k}-\boldsymbol{q}) /|\boldsymbol{k}-\boldsymbol{q}| \times \tilde{\psi}(q) \delta \tilde{J}_{\|}(k-q), \\
V_{m m \perp}(\boldsymbol{k}, \omega) \equiv-h_{c}^{-1} i \boldsymbol{k} \cdot \int_{q \Omega} \tilde{m}(q) \delta \tilde{\boldsymbol{J}}_{\perp}(k-q), \\
V_{m m \|}(\boldsymbol{k}, \omega) \equiv-h_{c}^{-1} i k \int_{q \Omega} \tilde{m}(q) \delta \tilde{J}_{\|}(k-q), \\
V_{\| \psi \psi}(\boldsymbol{k}, \omega) \equiv-i k T_{c} \beta_{2}^{-1} h_{c} \gamma_{0} \int_{q \Omega} \tilde{\psi}(q) \tilde{\psi}(k-q) .
\end{gathered}
$$

Since Eq. (A23) is decoupled from the other equations at linear level, we do not rewrite it as the matrix form.

Next, we calculate the bare propagator $G^{0}(\boldsymbol{k}, \omega)=\mathcal{M}^{-1}(\boldsymbol{k}, \omega)$. The inverse matrix is given as the transposed cofactor matrix divided by $\operatorname{det} \mathcal{M}$. The determinant reads

$$
\begin{aligned}
\operatorname{det} \mathcal{M}= & (-i \omega)^{3}+(-i \omega)^{2} k^{2}\left(\lambda D_{\psi} \chi_{0}^{-1}(\boldsymbol{k})+\nu_{0}^{l} h_{c}^{-1}\right)-i \omega k^{2}\left(C_{0}^{-1} h_{c} T_{c}+\chi_{0}^{-1}(\boldsymbol{k}) C_{\psi} C_{J} T_{c}\right) \\
& +k^{4} \lambda_{0} \chi_{0}^{-1} D_{\psi} C_{0}^{-1} h_{c} T_{c}-i \omega k^{4} \lambda_{0} \chi_{0}^{-1} D_{\psi}(\boldsymbol{k}) \nu_{0}^{l} h_{c}^{-1}
\end{aligned}
$$

Here, in the coefficient of $-i \omega k^{2}$, taking into account the behaviors after renormalization $[25,32]$, which are given as

$$
\begin{gathered}
C_{R}^{-1} \sim \xi^{-0.2}, \\
\chi_{R}^{-1}(\boldsymbol{k}) \sim \xi^{-2}+k^{2},
\end{gathered}
$$

we neglect $\chi_{0}^{-1}(\boldsymbol{k}) C_{\psi} C_{J} T_{c}$ by comparing with $C_{0}^{-1} h_{c} T_{c}$. Then, we can factorize the determinant as

$$
\begin{aligned}
\operatorname{det} \mathcal{M} \sim & \left(-i \omega+\lambda_{0}(\boldsymbol{k}) \chi_{0}^{-1}(\boldsymbol{k})\right) \\
& \times\left(-i \omega+i k c_{s}+\frac{1}{2} \nu_{0}^{l} h_{c}^{-1} k^{2}\right) \\
& \times\left(-i \omega-i k c_{s}+\frac{1}{2} \nu_{0}^{l} h_{c}^{-1} k^{2}\right),
\end{aligned}
$$

in the long-wavelength region. Here, we have defined

$$
\begin{gathered}
\lambda_{0}(\boldsymbol{k}) \equiv \lambda_{0} k^{2} D_{\psi}, \\
c_{s}^{2} \equiv C_{0}^{-1} h_{c} T_{c} .
\end{gathered}
$$

The diagonal components of the cofactor matrix $m$ read

$$
\begin{gathered}
m_{11} \sim\left(-i \omega+i k c_{s}+\frac{1}{2} \nu_{0}^{l} h_{c}^{-1} k^{2}\right) \\
\times\left(-i \omega-i k c_{s}+\frac{1}{2} \nu_{0}^{l} h_{c}^{-1} k^{2}\right), \\
m_{22}=(-i \omega)^{2}-i \omega k^{2} \lambda_{0} \chi_{0}(\boldsymbol{k}) D_{\psi} \nu_{0}^{l} h_{c}^{-1} \\
+k^{2} \chi_{0}^{-1}(\boldsymbol{k}) C_{\psi} C_{J} T_{c}+k^{4} \lambda_{0} \chi_{0}(\boldsymbol{k}) D_{\psi} \nu_{0}^{l} h_{c}^{-1}, \\
m_{33}=(-i \omega)\left(-i \omega+\lambda_{0}(\boldsymbol{k}) \chi_{0}^{-1}(\boldsymbol{k})\right),
\end{gathered}
$$

and the off-diagonal components are given by

$$
\begin{gathered}
m_{12}=k^{2} \chi_{0}^{-1}(\boldsymbol{k}) \beta_{2}^{-1} C_{J} T_{c}, \\
m_{13}=-k \omega \chi_{0}^{-1}(\boldsymbol{k}) C_{J} T_{c}, \\
m_{21}=-k^{2} C_{0}^{-1} h_{c} C_{\psi} \beta_{2} T_{c},
\end{gathered}
$$

$$
\begin{gathered}
m_{23}=-i k C_{0}^{-1} h_{c} \beta_{2} T_{c}\left(-i \omega+k^{2} \lambda_{0} \chi_{0}(\boldsymbol{k}) D_{\psi}\right), \\
m_{31}=k^{2} C_{0}^{-1} h_{c} C_{\psi} \beta_{2} T_{c}, \\
m_{32}=-i k \beta_{2}^{-1}\left(-i \omega+\lambda_{0}(\boldsymbol{k}) \chi_{0}^{-1}(\boldsymbol{k})\right) .
\end{gathered}
$$

Here, we neglect the off-diagonal components because they would not yield dominant contributions to the transport coefficients. Then, we obtain the bare propagator as

$$
G^{0}(\boldsymbol{k}, \omega)=\left(\begin{array}{ccc}
G_{\psi}^{0}(\boldsymbol{k}, \omega) & 0 & 0 \\
0 & G_{m}^{0}(\boldsymbol{k}, \omega) & 0 \\
0 & 0 & G_{\|}^{0}(\boldsymbol{k}, \omega)
\end{array}\right)
$$

with

$$
\begin{array}{r}
G_{\psi}^{0}(\boldsymbol{k}, \omega)=\frac{1}{-i \omega+\lambda(\boldsymbol{k}) \chi_{0}^{-1}(\boldsymbol{k})}, \\
G_{\|}^{0}(\boldsymbol{k}, \omega) \sim \\
\frac{1}{2}\left[\frac{1}{-i \omega+i k c_{s}+\frac{1}{2} \nu_{0}^{l} h_{c}^{-1} k^{2}}\right. \\
\left.+\frac{1}{-i \omega-i k c_{s}+\frac{1}{2} \nu_{0}^{l} h_{c}^{-1} k^{2}}\right] .
\end{array}
$$

$G_{m m}^{0}(\boldsymbol{k}, \omega)$ is not needed in later calculations. The bare propagator of $\delta \boldsymbol{J}_{\perp}$ is trivially given by

$$
G_{\perp}^{0}(\boldsymbol{k}, \omega)=\frac{1}{-i \omega+\eta_{0} k^{2} h_{c}^{-1}} .
$$


We finally arrive at the equations of motion as the selfconsistent form:

$$
\left(\begin{array}{c}
\tilde{\psi}(\boldsymbol{k}, \omega) \\
\tilde{m}(\boldsymbol{k}, \omega) \\
\delta \tilde{J}_{\|}(\boldsymbol{k}, \omega)
\end{array}\right)=\left(\begin{array}{c}
\tilde{\psi}^{0}(\boldsymbol{k}, \omega) \\
0 \\
\delta \tilde{J}_{\|}^{0}(\boldsymbol{k}, \omega)
\end{array}\right)+G^{0}(\boldsymbol{k}, \omega) \boldsymbol{V}(\boldsymbol{k}, \omega),
$$

and

$$
\delta \tilde{\boldsymbol{J}}_{\perp}(\boldsymbol{k}, \omega)=\delta \tilde{\boldsymbol{J}}_{\perp}^{0}(\boldsymbol{k}, \omega)+G_{\perp}^{0} \boldsymbol{V}_{\perp \psi \psi}(\boldsymbol{k}, \omega),
$$

where

$$
\begin{gathered}
\tilde{\psi}^{0}(\boldsymbol{k}, \omega)=G_{\psi}^{0}(\boldsymbol{k}, \omega) \alpha_{1}^{-1} \tilde{\theta}_{n}(\boldsymbol{k}, \omega), \\
\delta \tilde{J}_{\|}^{0}(\boldsymbol{k}, \omega)=G_{\|}^{0}(\boldsymbol{k}, \omega) \tilde{\theta}_{\|}(\boldsymbol{k}, \omega), \\
\delta \tilde{\boldsymbol{J}}_{\perp}^{0}(\boldsymbol{k}, \omega)=G_{\perp}^{0}(\boldsymbol{k}, \omega) \tilde{\boldsymbol{\theta}}_{\perp}(\boldsymbol{k}, \omega), \\
\boldsymbol{V}_{\perp \psi \psi}(\boldsymbol{k}, \omega)=-i T_{c} \mathcal{P}_{\perp}(\boldsymbol{k}) \cdot \int_{q \Omega} \boldsymbol{q} \chi_{0}^{-1}(\boldsymbol{q}) \\
\times \tilde{\psi}(q) \tilde{\psi}(k-q) .
\end{gathered}
$$

Here, $\tilde{\psi}^{0}(\boldsymbol{k}, \omega), \delta \tilde{J}_{\|}^{0}(\boldsymbol{k}, \omega)$ and $\delta \tilde{\boldsymbol{J}}_{\perp}^{0}$ are the bare variables that are the solutions without the nonlinear terms. Iterating Eqs. (A54) and (A55), we can obtain perturbative expansions about nonlinear interactions $\boldsymbol{V}$ and $\boldsymbol{V}_{\perp \psi \psi}$. We note that the first and third rows of Eq. (A54) are the equations of motion for the thermal and sound modes, respectively, while Eq. (A55) is for the viscous mode. We also stress that Eqs. (A51)-(A53) are the propagators of the thermal, sound and viscous modes, respectively.

Now, we calculate the two-body correlation of $\tilde{\psi}^{0}(\boldsymbol{k}, \omega)$ and $\delta \tilde{\boldsymbol{J}}_{\perp}^{0}(\boldsymbol{k}, \omega)$, which are needed in later calculations.

$$
\begin{aligned}
& \left\langle\tilde{\psi}^{0}\left(\boldsymbol{k}_{1}, \omega_{1}\right) \tilde{\psi}^{0}\left(\boldsymbol{k}_{2}, \omega_{2}\right)\right\rangle \\
& \quad=G_{\psi}^{0}\left(\boldsymbol{k}_{1}, \omega_{1}\right) G_{\psi}^{0}\left(\boldsymbol{k}_{2}, \omega_{2}\right) \alpha_{1}^{-2} \times\left\langle\tilde{\theta}\left(\boldsymbol{k}_{1}, \omega_{1}\right) \tilde{\theta}\left(\boldsymbol{k}_{2}, \omega_{2}\right)\right\rangle .
\end{aligned}
$$

Using the fluctuation-dissipation relation Eq. (53), we find

$$
\left\langle\tilde{\theta}\left(\boldsymbol{k}_{1}, \omega_{1}\right) \tilde{\theta}\left(\boldsymbol{k}_{2}, \omega_{2}\right)\right\rangle=2 \alpha_{1}^{2} \lambda_{0}(\boldsymbol{k})(2 \pi)^{d+1} \delta\left(k_{1}+k_{2}\right)
$$

and

$$
\begin{aligned}
& \left\langle\tilde{\psi}^{0}\left(\boldsymbol{k}_{1}, \omega_{1}\right) \tilde{\psi}^{0}\left(\boldsymbol{k}_{2}, \omega_{2}\right)\right\rangle \\
& \quad=\frac{2 \lambda_{0}\left(\boldsymbol{k}_{1}\right)}{\omega_{1}^{2}+\lambda_{0}^{2}\left(\boldsymbol{k}_{1}\right) \chi_{0}^{-2}\left(\boldsymbol{k}_{1}\right)} \times(2 \pi)^{d+1} \delta\left(k_{1}+k_{2}\right),
\end{aligned}
$$

where $\delta\left(k_{1}+k_{2}\right) \equiv \delta\left(\boldsymbol{k}_{1}+\boldsymbol{k}_{2}\right) \delta\left(\omega_{1}+\omega_{2}\right)$. By a similar calculation, we obtain

$$
\begin{aligned}
& \left\langle\delta \tilde{J}_{\perp}^{i}\left(\boldsymbol{k}_{1}, \omega_{1}\right) \delta \tilde{J}_{\perp}^{i}\left(\boldsymbol{k}_{2}, \omega_{2}\right)\right\rangle \\
& \quad=\frac{2 T_{c} \eta_{0}\left(\boldsymbol{k}_{1}\right)}{\omega_{1}^{2}+\eta_{0}^{2}\left(\boldsymbol{k}_{1}\right) h_{c}^{-2}}\left(\mathcal{P}_{\perp}\left(\boldsymbol{k}_{1}\right)\right)_{i j} \times(2 \pi)^{d+1} \delta\left(k_{1}+k_{2}\right),
\end{aligned}
$$

where $\eta_{0}(\boldsymbol{k})=\eta_{0} k^{2}$. For a later convenience, we define

$$
\begin{gathered}
C_{\psi}^{0}(\boldsymbol{k}, \omega)=\frac{2 \lambda_{0}(\boldsymbol{k})}{\omega^{2}+\lambda_{0}^{2}(\boldsymbol{k}) \chi_{0}^{-2}(\boldsymbol{k})}, \\
C_{\perp}^{0}(\boldsymbol{k}, \omega)=\frac{2 T_{c} \eta_{0}(\boldsymbol{k})}{\omega^{2}+\eta_{0}^{2}(\boldsymbol{k}) h_{c}^{-2}} .
\end{gathered}
$$

\section{APPENDIX B: RENORMALIZATION OF THE THERMAL AND VISCOUS DIFFUSION MODES}

Here, we first derive the RG equations for the thermal conductivity and the shear viscosity. Now, we note that the sound mode is not a genuine-relevant mode but a secondly mode that is strongly affected by order-parameter fluctuations but yields only a negligible feedback for the order parameters $[24,38]$. Then, we can neglect the sound mode for the minimal critical dynamics; however, the bulk viscosity is not renormalized in that case. Here, to first analyze the minimal dynamics, we neglect the secondary mode, which is renormalized in the next section. In that case, the equations of motion are given by

$$
\tilde{\psi}(\boldsymbol{k}, \omega)=\tilde{\psi}^{0}(\boldsymbol{k}, \omega)+G_{\psi}^{0}(\boldsymbol{k}, \omega) V_{\psi \psi \perp}(\boldsymbol{k}, \omega)
$$

and Eq. (A55). For a diagrammatic treatment, we denote the full and bare variables, the bare propagators and the bare correlation functions as Fig. 1. Then, we can represent the equations of motion (B1) and (A55) as Fig. 2.

For coarse gaining, we decompose the variables into the long- and short-wavelength components as

$$
\tilde{\psi}(\boldsymbol{k}, \omega)=\tilde{\psi}^{L}(\boldsymbol{k}, \omega)+\tilde{\psi}^{S}(\boldsymbol{k}, \omega)
$$

$$
\begin{aligned}
& \tilde{\psi}:=\tilde{\psi}^{0} \\
& G_{\psi}^{0}: \longrightarrow \quad C_{\psi}^{0}:-\mathbf{O} \\
& \delta \tilde{\boldsymbol{J}}_{\perp}: \square-\delta \tilde{\boldsymbol{J}}_{\perp}^{0}:--G_{\perp}^{0}:-\rightarrow-C_{\perp}^{0}:-\mathbf{0}-
\end{aligned}
$$

FIG. 1. Diagrams for the full and bare variables, the bare propagators, and the bare correlations. 

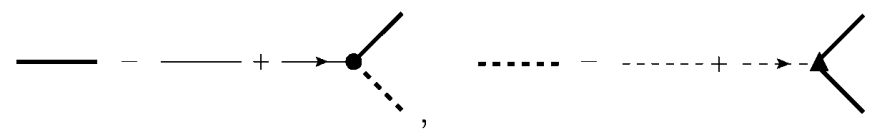

FIG. 2. Diagrams of the equations of motion for the thermal and viscous modes. The left- and right-hand side, respectively, denote Eqs. (B1) and (A55).

with

$$
\begin{gathered}
\tilde{\psi}^{L}(\boldsymbol{k}, \omega) \equiv \Theta(\Lambda-\delta \Lambda-k) \tilde{\psi}(\boldsymbol{k}, \omega), \\
\tilde{\psi}^{S}(\boldsymbol{k}, \omega) \equiv \Theta(k-\Lambda-\delta \Lambda) \tilde{\psi}(\boldsymbol{k}, \omega),
\end{gathered}
$$

where $\Theta(x)$ is a step function; i.e., the wave number is decomposed into $0<k<\Lambda-\delta \Lambda$ and $\Lambda-\delta \Lambda<k<\Lambda$. Hereafter, quantities with the suffixes $L$ and $S$ are supposed to be decomposed as above. To average over the $\tilde{\psi}^{0 S}$ and $\delta \tilde{\boldsymbol{J}}_{\perp}^{0 S}$, we must solve the equation of motion about them. Here, we solve the equations of motion to second order in the nonlinear interactions and average over $\tilde{\psi}^{0 S}$ and $\delta \tilde{\boldsymbol{J}}_{\perp}^{0 S}$. Then, we find the coarse-grained equation of motion for $\psi$, which is diagrammatically given by Fig. 3. The last two terms in Fig. 3 represent nonlinear interactions being of third order, and can be neglected. Furthermore, the fifth term vanishes due to the relation between the step and delta functions in the loop integral. Introducing the self energy $\Sigma_{\psi \psi}$, which is graphically represented in Fig. 4, we can write the coarse-grained equation of motion for $\psi$ as

$$
\begin{aligned}
\tilde{\psi}^{L}(\boldsymbol{k}, \omega)= & \tilde{\psi}^{0 L}(\boldsymbol{k}, \omega)+G_{\psi}^{0 L}(\boldsymbol{k}, \omega) V_{\psi \psi \perp}^{L}(\boldsymbol{k}, \omega) \\
& +\tilde{\psi}^{L}(\boldsymbol{k}, \omega) G_{\psi}^{0 L}(\boldsymbol{k}, \omega) \Sigma_{\psi \psi}(\boldsymbol{k}, \omega) .
\end{aligned}
$$

The self energy is given by

$\Sigma_{\psi \psi}(\boldsymbol{k}, \omega)=-T_{c} h_{c}^{-1} k^{2} \chi_{0}^{-1}(\boldsymbol{k})$

$$
\times \int_{q} \frac{(\hat{\boldsymbol{k}} \cdot \mathcal{P}(\boldsymbol{k}-\boldsymbol{q}) \cdot \hat{\boldsymbol{k}}) \chi_{0}(\boldsymbol{q})}{-i \omega+\lambda_{0}(\boldsymbol{q}) \chi_{0}^{-1}(\boldsymbol{q})+\eta_{0}(\boldsymbol{k}-\boldsymbol{q}) h_{c}^{-1}},
$$

where $\eta_{0}(\boldsymbol{k})=\eta_{0} k^{2}$. Solving Eq. (B5) about $\tilde{\psi}^{L}$, we have

$$
\begin{aligned}
\tilde{\psi}^{L}= & {\left[\left(G_{\psi}^{0 L}\right)^{-1}-\Sigma_{\psi \psi}\right]^{-1} \alpha_{1}^{-1} \tilde{\theta}_{n} } \\
& +\left[\left(G_{\psi}^{0 L}\right)^{-1}-\Sigma_{\psi \psi}\right]^{-1} V_{\psi \psi \perp}^{L},
\end{aligned}
$$

where we have used Eq. (A56). Introducing renormalized variables as

$$
\begin{gathered}
\left(G_{\psi R}\right)^{-1}(\boldsymbol{k}, \omega)=\left(G_{\psi}^{0 L}\right)^{-1}(\boldsymbol{k}, \omega)-\Sigma_{\psi \psi}(\boldsymbol{k}, \omega), \\
\tilde{\psi}_{R}^{0 L}(\boldsymbol{k}, \omega)=G_{\psi R}(\boldsymbol{k}, \omega) \alpha_{1}^{-1} \tilde{\theta}_{n}(\boldsymbol{k}, \omega),
\end{gathered}
$$
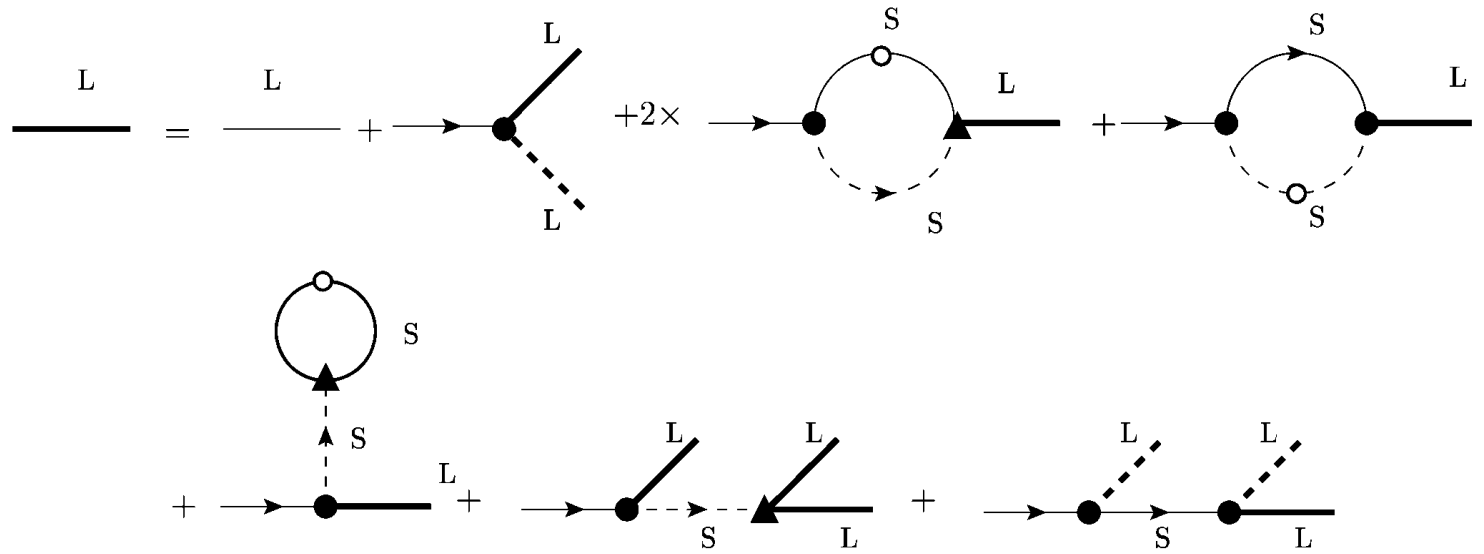

FIG. 3. Diagrams for the coarse-grained equation of motion for $\psi$. The letters, $L$ and $S$, respectively, denote the long- and shortwavelength components (see the text below Eq. (B4)).

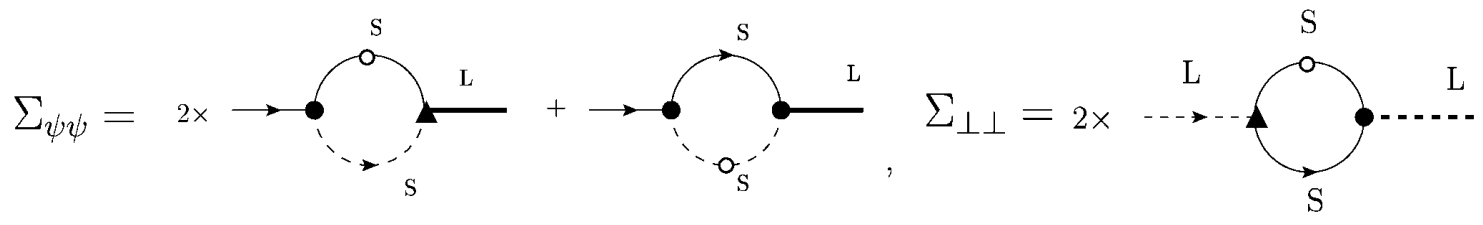

FIG. 4. Diagrams for the self energies. 
we can rewrite Eq. (A56) as the renormalized equation of motion:

$$
\tilde{\psi}^{L}=\tilde{\psi}_{R}^{0 L}(\boldsymbol{k}, \omega)+G_{\psi R}(\boldsymbol{k}, \omega) V_{\psi \psi \perp}^{L} .
$$

We now require that the renormalized propagator has the same form as the bare one:

$$
\left(G_{\psi R}\right)^{-1}(\boldsymbol{k}, \omega)=-i \omega+\lambda_{R} D_{\psi} k^{2} \chi_{0}^{-1}(\boldsymbol{k}),
$$

where $\lambda_{R}$ is the renormalized thermal conductivity. That is, we require that the only transport coefficients are explicitly renormalized. The small correction for the thermal conductivity $\delta \lambda \equiv \lambda_{R}-\lambda_{0}$ reads

$$
\begin{aligned}
\delta \lambda & =-\lim _{k, \omega \rightarrow 0}\left[\left(D_{\psi} k^{2} \chi_{0}(\boldsymbol{k})\right)^{-1} \Sigma_{\psi \psi}(\boldsymbol{k}, \omega)\right], \\
& =\frac{T_{c}}{h_{c} D_{\psi}} \int_{q} \frac{(\hat{\boldsymbol{k}} \cdot \mathcal{P}(\boldsymbol{q}) \cdot \hat{\boldsymbol{k}}) \chi_{0}(\boldsymbol{q})}{\lambda_{0}(\boldsymbol{q}) \chi_{0}^{-1}(\boldsymbol{q})+\eta_{0}(\boldsymbol{q}) h_{c}^{-1}} .
\end{aligned}
$$

We approximate the denominator and the numerator as

$$
\begin{gathered}
\lambda_{0}(\boldsymbol{q}) \chi_{0}^{-1}(\boldsymbol{q})+\eta_{0}(\boldsymbol{q}) h_{c}^{-1} \sim \eta_{0}(\boldsymbol{k}) h_{c}^{-1}, \\
\chi_{0}^{-1}(\boldsymbol{q})=r_{0}+q^{2} \sim q^{2},
\end{gathered}
$$

near the $C P[28]$. Then, we find

$$
\begin{aligned}
\delta \lambda & \sim \frac{T_{c}}{D_{\psi} \eta_{0}} \int \frac{d \Omega_{d}}{(2 \pi)^{d}}(\hat{\boldsymbol{k}} \cdot \mathcal{P}(\boldsymbol{q}) \cdot \hat{\boldsymbol{k}}) \int_{\Lambda-\delta \Lambda}^{\Lambda} d q q^{d-5} \\
& =-\frac{T_{c}}{D_{\psi} \eta_{0}} \int \frac{d \Omega_{d}}{(2 \pi)^{d}}(\hat{\boldsymbol{k}} \cdot \mathcal{P}(\boldsymbol{q}) \cdot \hat{\boldsymbol{k}}) \Lambda^{d-5} \delta \Lambda,
\end{aligned}
$$

where $d \Omega_{d}$ is the solid angle in the space dimension $d$. Therefore, we obtain the RG equation for the thermal conductivity:

$$
-\Lambda \frac{\partial \lambda}{\partial \Lambda}=\frac{T_{c}}{D_{\psi} \eta(\Lambda)} \int \frac{d \Omega_{d}}{(2 \pi)^{d}}(\hat{\boldsymbol{k}} \cdot \mathcal{P}(\boldsymbol{q}) \cdot \hat{\boldsymbol{k}}) \Lambda^{d-4},
$$

where $\eta_{0}$ is rewritten as $\eta(\Lambda)$. For the space dimensions, $d=4-\epsilon$, the angle integral is given by

$$
\int \frac{d \Omega_{4}}{(2 \pi)^{4}}(\hat{\boldsymbol{k}} \cdot \mathcal{P}(\boldsymbol{q}) \cdot \hat{\boldsymbol{k}})=\frac{3}{4} K_{4},
$$

where $K_{4}$ is the surface area of a unit sphere in 4 dimensions divided by $(2 \pi)^{4}$. The RG equation in $4-\epsilon$ dimensions reads

$$
-\Lambda \frac{\delta \lambda}{\delta \Lambda}=\frac{3}{4} f(\Lambda) \lambda(\Lambda),
$$

where we have introduced

$$
f(\Lambda) \equiv \frac{T_{c} K_{4}}{D_{\psi} \eta(\Lambda) \lambda(\Lambda) \Lambda^{\epsilon}},
$$

for a later convenience.

By making coarse-graining of the viscous mode with a similar procedures as above, we obtain a small correction for the shear viscosity:

$$
\delta \eta=-\lim _{k, \omega \rightarrow 0}\left[\left(k^{2} h_{c}^{-1}(d-1)\right)^{-1} \sum_{i}\left(\Sigma_{\perp \perp}(\boldsymbol{k}, \omega)\right)_{i i}\right],
$$

where $\left(\Sigma_{\perp \perp}(\boldsymbol{k}, \omega)\right)_{i j}$ is the self energy for the viscous mode and given by

$$
\left(\Sigma_{\perp \perp}(\boldsymbol{k}, \omega)\right)_{i j}=-T_{c} h_{c}^{-1} \int_{q} \chi_{0}(\boldsymbol{k}-\boldsymbol{q})\left(\mathcal{P}_{\perp}(\boldsymbol{k}) \cdot \boldsymbol{q}\right)_{i} q_{j} \times \frac{\chi_{0}^{-1}(\boldsymbol{q})-\chi_{0}^{-1}(\boldsymbol{k}-\boldsymbol{q})}{-i \omega+\lambda_{0}(\boldsymbol{q}) \chi_{0}^{-1}(\boldsymbol{q})+\lambda(\boldsymbol{k}-\boldsymbol{q}) \chi_{0}^{-1}(\boldsymbol{k}-\boldsymbol{q})},
$$

which is graphically represented as Fig. 4. In the space dimension $d=4-\epsilon$, we find the RG equation for the shear viscosity

$$
-\Lambda \frac{\partial \eta(\Lambda)}{\partial \Lambda}=\frac{1}{24} f(\Lambda) \eta(\Lambda)
$$

where the prefactor $1 / 24$ comes from the angular integral in Eq. (B21) and the factor $(d-1)^{-1}$ in Eq. (B20).

Differentiating Eq. (B19) about $\Lambda$, we have the RG equation for $f(\Lambda)$

$$
-\Lambda \frac{\partial f(\Lambda)}{\partial \Lambda}=\left(\epsilon-\frac{19}{24} f(\Lambda)\right) f(\Lambda)
$$

\section{APPENDIX C: RENORMALIZATION OF THE SOUND MODE}

Next, let us make a coarse-graining of the sound mode for the renormalized bulk viscosity. Because a feedback from the sound mode is neglected, we must renormalize the mode with a method separating relevant and secondly modes [38]. Here, we take the method developed by Onuki $[21,25]$, in which $R G$ equations are derived from fluctuation-dissipation relations.

Now, we consider the equation of motion for the sound mode, (A22):

$$
\begin{aligned}
-i \omega \delta \tilde{J}_{\|}= & -i k T_{c}\left[\chi_{0}^{-1}(\boldsymbol{k}) C_{J} \tilde{\psi}+C_{0}^{-1} \beta_{2} h_{c} \tilde{m}\right. \\
& \left.+\beta_{2}^{-1} h_{c} \gamma_{0} \int_{q \Omega} \tilde{\psi}(q) \tilde{\psi}(k-q)\right] \\
& -k^{2} \nu_{0}^{l} h_{c}^{-1} \delta \tilde{J}_{\|}+\tilde{\theta}_{\|}^{0},
\end{aligned}
$$


where the noise term $\tilde{\theta}_{\|}^{0}$ satisfies the fluctuation-dissipation relation:

$$
\left\langle\tilde{\theta}_{\|}^{0}\left(\boldsymbol{k}_{1}, \omega_{1}\right) \tilde{\theta}_{\|}^{0}\left(\boldsymbol{k}_{2}, \omega_{2}\right)\right\rangle=2 T_{c} k_{1}^{2} \nu_{0}^{l} \times(2 \pi)^{d+1} \delta\left(k_{1}+k_{2}\right) .
$$

Since $\delta \boldsymbol{J}_{\|}$is a conserved density projected onto $\hat{\boldsymbol{k}}$, we can rewrite Eq. (C1) as

$$
-i \omega \delta \tilde{J}_{\|}(\boldsymbol{k}, \omega)=i \boldsymbol{k} \cdot \tilde{\Pi}(\boldsymbol{k}, \omega) \cdot \hat{\boldsymbol{k}},
$$

where $\tilde{\Pi}_{i j}$ is the stress tensor. If we take $z$ direction as $\hat{\boldsymbol{k}}$, $\tilde{\Pi}_{z z}$ reads

$$
\begin{aligned}
\tilde{\Pi}_{z z}(\boldsymbol{k}, \omega)= & -T_{c}\left[\chi_{0}^{-1}(\boldsymbol{k}) C_{J} \tilde{\psi}(\boldsymbol{k}, \omega)+C_{0}^{-1} \beta_{2} h_{c} \tilde{m}(\boldsymbol{k}, \omega)\right. \\
& \left.+\beta_{2}^{-1} h_{c} \gamma_{0} \int_{q \Omega} \tilde{\psi}(q) \tilde{\psi}(k-q)\right] \\
& +i k \nu_{0}^{l} h_{c}^{-1} \delta \tilde{J}_{\|}(\boldsymbol{k}, \omega)+\tilde{\pi}_{z z}^{0}(\boldsymbol{k}, \omega), \quad(\mathrm{C} 4)
\end{aligned}
$$

where $\tilde{\pi}_{i j}^{0}(\boldsymbol{k}, \omega)$ is the random-stress tensor coming from microscopic process and satisfies the relation, $i \boldsymbol{k}$. $\tilde{\pi}^{0}(\boldsymbol{k}, \omega) \cdot \hat{\boldsymbol{k}}=\tilde{\theta}_{\|}^{0}(\boldsymbol{k}, \omega)$.

We now consider how Eq. (C1) is affected by the coarsegraining procedure. In the coarse-graining procedure, the variables, $\tilde{\psi}^{S}, \tilde{m}^{S}$ and $\delta \tilde{\boldsymbol{J}}^{S}$ are eliminated from Eq. (C1). The eliminated variables do not disappear from the equation of motion but are implicitly contained in the noise term. In other words, we convert the macroscopic process in the wave number shell $\Lambda-\delta \Lambda<k<\Lambda$ into the microscopic process. In this procedure, the noise term is implicitly renormalized as follows:

$$
\tilde{\theta}_{\|}^{R}(\boldsymbol{k}, \omega)=\tilde{\theta}_{\|}^{0}(\boldsymbol{k}, \omega)+\tilde{\theta}_{\|}^{\mathrm{Macro}}(\boldsymbol{k}, \omega),
$$

where

$$
\begin{aligned}
& \tilde{\theta}_{\|}^{\mathrm{Macro}}(\boldsymbol{k}, \omega) \equiv i \boldsymbol{k} \cdot \tilde{\pi}^{\mathrm{Macro}}(\boldsymbol{k}, \omega) \cdot \hat{\boldsymbol{k}}, \\
& \tilde{\pi}_{z z}^{\mathrm{Macro}}(\boldsymbol{k}, \omega) \equiv-T_{c}\left[\chi_{0}^{-1}(\boldsymbol{k}) C_{J} \tilde{\psi}^{S}+C_{0}^{-1} \beta_{2} h_{c} \tilde{m}^{S}\right. \\
&\left.+\beta_{2}^{-1} h_{c} \gamma_{0} \int_{q \Omega} \tilde{\psi}^{S}(q) \tilde{\psi}^{S}(k-q)\right] \\
&+i k \nu_{0}^{l} h_{c}^{-1} \delta \tilde{J}_{\|}^{S}, \\
& \sim-T_{c} \beta_{2}^{-1} h_{c} \gamma_{0} \int_{q \Omega} \tilde{\psi}^{S}(q) \tilde{\psi}^{S}(k-q),
\end{aligned}
$$

where we neglect the linear terms in Eq. (C7) that is irrelevant for the following argument. The new term $\tilde{\theta}_{\|}^{\text {Macro }}(\boldsymbol{k}, \omega)$, being due to the coarse-graining, contributes the transport coefficient through the fluctuation-dissipation relation:

$$
\begin{aligned}
& \left\langle\tilde{\theta}_{\|}^{\text {Macro }}\left(\boldsymbol{k}_{1}, \omega_{1}\right) \tilde{\theta}_{\|}^{\text {Macro }}\left(\boldsymbol{k}_{2}, \omega_{2}\right)\right\rangle \\
& \quad=2 T_{c} k_{1}^{2} \delta \nu^{l}\left(\boldsymbol{k}_{1}, \omega_{1}\right)(2 \pi)^{d+1} \delta\left(k_{1}+k_{2}\right),
\end{aligned}
$$

where we have assumed that the renormalized equation of motion has the same form as Eq. (C1). We note that this assumption is equivalent to the requirement below Eq. (B10). Now, we calculate the left-hand side in Eq. (C9):

$$
\left\langle\tilde{\theta}_{\|}^{\mathrm{Macro}}\left(\boldsymbol{k}_{1}, \omega_{1}\right) \tilde{\theta}_{\|}^{\mathrm{Macro}}\left(\boldsymbol{k}_{2}, \omega_{2}\right)\right\rangle=-k_{1} k_{2}\left(T_{c} h_{c} \beta_{2}^{-1}\right)^{2} \gamma_{0}^{2} \times \int_{q_{1} \Omega_{1} q_{2} \Omega_{2}}\left\langle\tilde{\psi}^{S}\left(q_{1}\right) \tilde{\psi}^{S}\left(k_{1}-q_{1}\right) \tilde{\psi}^{S}\left(q_{2}\right) \tilde{\psi}^{S}\left(k_{2}-q_{2}\right)\right\rangle .
$$

Approximating the variable by the bare one, $\tilde{\psi}^{S} \sim \tilde{\psi}^{0 S}$, we find

$$
\left\langle\tilde{\theta}_{\|}^{\mathrm{Macro}}\left(\boldsymbol{k}_{1}, \omega_{1}\right) \tilde{\theta}_{\|}^{\mathrm{Macro}}\left(\boldsymbol{k}_{2}, \omega_{2}\right)\right\rangle=(2 \pi)^{d+1} \delta\left(k_{1}+k_{2}\right) \times 2 k_{1}^{2}\left(T_{c} h_{c} \beta_{2}^{-1}\right)^{2} \gamma_{0}^{2} \int_{q \Omega} C_{\psi}^{0 S}(q) C_{\psi}^{0 S}\left(k_{1}-q\right),
$$

where we have used Eq. (A62) and neglected a term corresponding to a disconnected diagram. Then, comparing with Eq. (C9), we obtain the correction to the longitudinal-kinetic viscosity:

$$
\delta \nu^{l}(\boldsymbol{k}, \omega)=T_{c} \beta_{2}^{-2} h_{c}^{2} \gamma_{0}^{2} \int_{q \Omega} C_{\psi}^{0 S}(q) C_{\psi}^{0 S}(k-q) .
$$

We are not interested in the frequency-dependent or wave number-dependent bulk viscosity, and then take the limit $k$, $\omega \rightarrow 0$ :

$$
\delta \nu^{l} \equiv \lim _{k, \omega \rightarrow 0} \delta \nu^{l}(\boldsymbol{k}, \omega)=T_{c} \beta_{2}^{-2} h_{c}^{2} \gamma_{0}^{2} \int_{q \Omega}\left(C_{\psi}^{0 S}(q)\right)^{2} .
$$

After the integration, we find the RG equation for longitudinal kinetic viscosity:

$$
-\Lambda \frac{\partial \nu^{l}(\Lambda)}{\partial \Lambda}=\frac{T_{c} h_{c}^{2} K_{4}}{\beta_{2}^{2} D_{\psi}} \gamma^{2}(\Lambda) \lambda^{-1}(\Lambda) \Lambda^{-\epsilon-4}
$$

where we have rewritten the static parameter $\gamma_{0}$ as $\gamma(\Lambda)$ to denote its cutoff dependence as mentioned in the text. The asymptotic behavior obtained from this RG equation is different from the shear viscosity's behavior, so we replace above $R G$ equation as 


$$
-\Lambda \frac{\partial \zeta(\Lambda)}{\partial \Lambda}=\frac{T_{c} h_{c}^{2} K_{4}}{\beta_{2}^{2} D_{\psi}} \gamma^{2}(\Lambda) \lambda^{-1}(\Lambda) \Lambda^{-\epsilon-4} .
$$

Although, by this method, we could more easily obtain the RG equations for the thermal conductivity and shear viscosity, we have taken the diagrammatic method for an instructive purpose.
[1] M. Asakawa and K. Yazaki, Nucl. Phys. A504, 668 (1989); A. Barducci, R. Casalbuoni, G. Pettini, and R. Gatto, Phys. Lett. B 231, 463 (1989); Phys. Rev. D 49, 426 (1994); As review articles, see, M. A. Stephanov, Prog. Theor. Phys. Suppl. 153, 139 (2004); Int. J. Mod. Phys. A 20, 4387 (2005).

[2] M. Kitazawa, T. Koide, T. Kunihiro, and Y. Nemoto, Prog. Theor. Phys. 108, 929 (2002); Z. Zhang, K. Fukushima, and T. Kunihiro, Phys. Rev. D 79, 014004 (2009); Z. Zhang and T. Kunihiro, Phys. Rev. D 80, 014015 (2009).

[3] N. Yamamoto, M. Tachibana, T. Hatsuda, and G. Baym, Phys. Rev. Lett. 97, 122001 (2006); Phys. Rev. D 76, 074001 (2007).

[4] M. G. Alford, J. A. Bowers, and K. Rajagopal, Phys. Rev. D 63, 074016 (2001).

[5] E. Nakano and T. Tatsumi, Phys. Rev. D 71, 114006 (2005); S. Maedan, Prog. Theor. Phys. 123, 285 (2010); M. Buballa and D. Nickel, Acta Phys. Pol. 3, 523 (2010); S. Carignano, D. Nickel, and M. Buballa, Phys. Rev. D 82, 054009 (2010); T. Kojo, Y. Hidaka, L. McLerran, and R. D. Pisarski, Nucl. Phys. A843, 37 (2010).

[6] P. de Forcrand and O. Philipsen, J. High Energy Phys. 01 (2007) 077.

[7] K. Fukushima, Phys. Rev. D 78, 114019 (2008).

[8] M. A. Stephanov, K. Rajagopal, and E. V. Shuryak, Phys. Rev. Lett. 81, 4816 (1998); Phys. Rev. D 60, 114028 (1999).

[9] T. Kunihiro, Phys. Lett. B 271, 395 (1991).

[10] Y. Hatta and T. Ikeda, Phys. Rev. D 67, 014028 (2003).

[11] H. Fujii, Phys. Rev. D 67, 094018 (2003); H. Fujii and M. Ohtani, Phys. Rev. D 70, 014016 (2004); Prog. Theor. Phys. Suppl. 153, 157 (2004); H. Fujii and N. Tanji, J. Phys. G 35, 104060 (2008).

[12] D. T. Son and M. A. Stephanov, Phys. Rev. D 70, 056001 (2004).

[13] M. A. Stephanov, Phys. Rev. Lett. 102, 032301 (2009).

[14] See, for example, H. Haken, Rep. Prog. Phys. 52, 515 (1989).

[15] F. Karsch, D. Kharzeev, and K. Tuchin, Phys. Lett. B 663, 217 (2008).

[16] P. Romatschke and D. T. Son, Phys. Rev. D 80, 065021 (2009).
[17] G. D. Moore and O. Saremi, J. High Energy Phys. 09 (2008) 015.

[18] C. Sasaki and K. Redlich, Nucl. Phys. A832, 62 (2010).

[19] H. Mori and H. Fujisaka, Prog. Theor. Phys. 49, 764 (1973).

[20] K. Kawasaki, Ann. Phys. (N.Y.) 61, 1 (1970).

[21] A. Onuki, Phase Transition Dynamics (Cambridge University Press, Cambridge, England, 2007).

[22] G.F. Mazenko, Nonequilibrium Statistical Mechanics (WiLEY-VCH, Weinheim, Germany, 2006).

[23] H. Mori, Prog. Theor. Phys. 33, 423 (1965).

[24] Y. Minami and T. Kunihiro, Prog. Theor. Phys. 122, 881 (2010).

[25] A. Onuki, Phys. Rev. E 55, 403 (1997), and references therein.

[26] L.D. Landau and E.M. Lifshitz, Fluid Mechanics (Pergamon, New York, 1959).

[27] K. Kawasaki, Nonequilibrium and Phase Transition (Asakura Syoten, Tokyo, 2000), in Japanese.

[28] E. D. Siggia, B. I. Halperin, and P. C. Hohenberg, Phys. Rev. B 13, 2110 (1976).

[29] K. Kawasaki and J. Gunton, Phys. Rev. B 13, 4658 (1976).

[30] B. I. Halperin, P. C. Hohenberg, and E. D. Siggia, Phys. Rev. B 13, 1299 (1976).

[31] B. I. Halperin and P. C. Hohenberg, Phys. Rev. Lett. 19, 700 (1967); Phys. Rev. 177, 952 (1969); P. C. Hohenberg and B. I. Halperin, Rev. Mod. Phys. 49, 435 (1977).

[32] B. I. Halperin, P. C. Hohenberg, and S. Ma, Phys. Rev. B 10, 139 (1974); 13, 4119 (1976).

[33] C. Nonaka and M. Asakawa, Phys. Rev. C 71, 044904 (2005); C. E. Aguiar, T. Kodama, T. Koide, and Y. Hama, Braz. J. Phys. 37, 95 (2007).

[34] Y. Akamatsu, T. Hatsuda, and T. Hirano, Phys. Rev. C 79, 054907 (2009).

[35] W. Israel, Ann. Phys. (N.Y.) 100, 310 (1976); W. Israel and J. M. Stewart, Ann. Phys. (N.Y.) 118, 341 (1979).

[36] W. A. Hiscock and L. Lindblom, Phys. Rev. D 31, 725 (1985).

[37] M. Kitazawa, T. Koide, T. Kunihiro, and Y. Nemoto, Phys. Rev. D 65, 091504 (2002); Prog. Theor. Phys. 114, 117 (2005).

[38] D. M. Kroll and J. M. Ruhland, Phys. Lett. A 80, 45 (1980). 